\title{
Zum Ursprung und Wesen der reduzierten Vokale im Mordwinischen
}

1. Vom phonetischen standpunkt aus gesehen hat das Mokscha-Mordwinische zwei reduzierte Vokale, das palatale a und das velare $\hat{\partial}$. Nach den uns zur Verfügung stehenden, relativ spärliche'l Quellen ist ihr Auftreten in den Dialekten meinheitlich, was auch aus den Definitionen dor diesbezüglichen Vrephältnisse hervorount. In der »Grammatika mordorskich jazykor, Fonotika i morfologijan heisst es S. 21, das a trete nach monillierten Konsonanten auf und das $\hat{\partial}$ sonst. Andererseits kann -sowohl in der ersten Silbe als auch weiter hinten im Wort -. auch die (Qualität eines nachfolgenden Konsonanten den Vokal dahingehend beeinflussen, dass vor einem monillierten Konsonanten a ansgesprochen wird, vor nichtmouillierten Konsonanten dagegen $\hat{\partial}$, s. S. I. Lipatov SFU $\bigvee 178$, vgl. auch H. PAAsoNex MSFOU XXII 86. I'ber den allgemeinen Charakter der sekundären Konsonantenmouillierung im Mordwinischen sagt Paasonen folgendes: "Vor den vorder'n vollkommen gebildeten rocalen sind alle consonanten im mordvinischen mouillirt. Es liegt in der natur der laute, dass die monillirung an den dentalen consonanten am bemerkbarsten hervortritt. Yor den vorderen reducirten vocalen macht sich die mouillirung nur an ihnen, nicht aber an den labialen (und palatalen) dem gehörs kund", s. a.il.). 48. Paasonen weist hin auf den im Mokscha nach I sabialen und Palatalen begegnenden paradigmatischen Wechsel $\partial \sim \hat{\partial}$, der durch die Qualität des nachfolgenden Konsonanten hrrvorgerufen wird, z.B. ṕingar (ièll. pingâxa Prolat. 'Zeit', s. a.a.(1. 86. Er führt ferner den interdialektalen Wechsel an (Selišče) vârga's 'Wolf', sembân Gen. 'all' 〜 (Pšenevo) vaŕga·s, śemban, s. a.a.(). 97, 86. Im Lichte dieser Belege würde im erstern I Dialekttyp dio Rogel wâ nilch pinem nichtmouillierten 
Konsonanten" und im letzteren "d ror rinem monillierten Konsonanten" herrschen.

Bei Paasonen und J. Juhísz finden sich jedoch auch zahlreiche Belege für ein palatales a in Mokscha, das von allen oben angeführten Regeln abweicht, indem as zwischen zwei nichtmouillierten Konsonimten steht. Beide Verfasser haben z.B. kolgams 'triefen, rinnen. sickern; leck sein, lecken', andererseits aber z.B. solgâms 'zumachen, verschliessen'. Dir normative Orthographie des Mokscha kennt keinen solchen Enterschied in der Qualität des Vokals der zweiten silbe, somdern danach

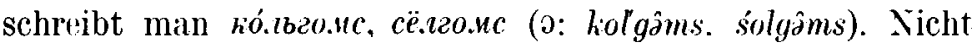
alle Dialekte scheinen in dieser Hinsicht den grleichen Stand zu vertreten. So hat Paasonen rtwa musliams (Mordw. Chr. 97) 〜 muśliams (MSFOu XXII 81) 'walschen' alufgezeichnet, welche' Formen auch bei Juhász in interdialektalem Wechsel reschoinen. Die Orthographie der mokschanischen sichriftsplache notiert lediglich die Form .nýcbnonc $(=$ muśliâms). Ein solches eigenartig wirkendes Verhältnis der Schriftsprache wie sáu..ue Nom. ва́.ймонь Gen. 'Seele; Atem', bei Juhász rajrí vajmân, geht offensichtlich zurück auf die Form rujne rajmań und erklärt sich aus Paasonens oben zitiertor Rugel "mouillierter Labial vor einem vorderen Vollvokil, nichtmouillierter Labial vor einem vorderen reduzierten Vokilly. Wir kömnen uns hier nicht eingehender nit den Voraussetzungen für das Auftreten der Vokale a und â beschäftigum; mehr Angabe'n dazu - wenn auch nicht erschöpfender Art - finden sich bei Juhás\% in der Einleitung zu seinem Wörterbuch "Moksa-mordvin szíjegyzék" S. 10-13. Die Frage nach den reduzierten Vokalen im Mordwinischen würde als Ganzes eine Spezialuntersuchung verdienen, wozu rin umfassendes und phonetisch zuverlässiges Material notwendi⿺r wäre. Berücksichtigt werden müssen natürlich auch die Erza-Dialekte, in denen reduzierto Vokale auftreten. Die folgende Darstellung will eim Einführung geben in die allgemeinen Prinzipien einer solchen Untersuchung.

2.1.1. Wir beschäftigen uns eingangs mit den reduzierten Vokalen der ers te n sil be.

2.1.2. In einigen Wörter'n der Mokschil-Dialekte hat sich das palatale a ans einem urmurdw. e rntwickelt, das in irondenem 
anderen Wort oder in einer Form gleicher Herkunft erhalten ist. Z.B. maźa·ra 'wieviel', maźa·rda 'wann' < me·źc 'was' < vormordw. *mi-; dial. kamga·ftâva 'zwölf' ( K'emgaftâva, E k'emgavtovo usw.) < kemań 'zehn'. Das Wort kamga'ftâra ist ein Kompositum mit der zweiten Komponente kafta, kaftz-' 'zwei'. Aller Wahrscheinlichkeit nach sind auch maźa ra und maźa rda Komposita, s. A. Raun ÕEST XXX 589-595. Das $a$ in de? zweiten Silbe dieser Wörter stand also als Vokal in der crsten Silbe eines einzelnen Wortes und hat sich seinen Hauptdruck bewahrt. Die Wörter mit dem Wandel $e>\theta$ haben sich im Sprachgefühl von Komposita zu Ableitungen verwandelt. Durch die Akzentregel im Mokscha liegt der Hauptdruck generell auf der ersten Silbe, in dem Fall jedoch auf der zweiten, wenn als Sonant in der letztgenannten Silbe ein urmordw. a steht und in der ersten Silbe $u, i$ oder ein reduzierter Vokal. (In einigen Dialekten ist das a der zweiten Silbe im Wortauslaut stets drucklos.) Da vor der hauptbetonten zweiten Silbe kein $e$ auftreten kann, hat sich in den Typen maźa ra, kamga·ftâva dẹ Wandel $e>a$ vollziehen müssen, vgl. Paasonen, a.a.O. 98. In der Variante kemgaftâva liegt der Hauptakzent auf der ersten Silbe, aus welchem Grunde das e erhalten bleiben konnte. Auch M śńarra 'soviel' weist Vokalreduktion aluf. Die Erscheinung erklärt sich hier sonst wie oben, doch stellt sich die Frage. ob $e$ oder $\varepsilon(<a)$ Gegenstand der Rrduktion wurde. Beidr Ausgangsformen scheinen auf den ersten Blick möglich, da in dem als erste Komponente des betr. Wortes stehenden Pronomen śs, śa- 'jener; dieser da; der, derjenige' ein Vokalwechsel erwähnter Art begegnet, z.B. salia $\sim$ selk 'derselbe, ein und derselbe; ebender; einzig'. Zweifellos ist jedoch anch hier auszugehen von $e$, das in dem diesbezüglichen Pronomen urmordwinisch ist (vgl. E śe, fi. se), während sich die Stammvariante $\dot{s} a-(>$ in bestimmten Fällen ś) als Erofobnis mokschanischer Sonderentwicklung erweist.

Gesichert wird die Schlussfolgerung, der Vokal der ersten Silbe in den Wörtern maźa·ra (maźa·rda) und sańa·ra gehe auf ein früheres $e$ zurück, durch den Sachverhalt, dass der in Frage stehende Reduktionsprozess bereits urmordwinisch sein muss. Dats wird bewiesen durch die entsprechenden Erza-For- 
Inen: (Kažlỵtkil) męźr ra, (Kiljajevo) miźara (hier $i<\imath)$, (Ma-

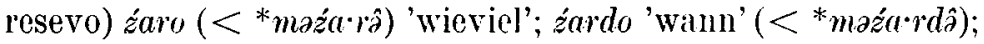
śnaro, śnaro 'soviel' (<*'sana·râ). Auch im Erza steht also in diesen Wörtern ein reduzirrter Vokil, der - weil unbetont dialektweise auch völlig hat schwinden kömmen, rọl. Paasonen. i.a.O. Die Vokalreduktion der Form kamga ftâca stammt aus einer späteren Zeit und beschränkt sich nur anf einign" MokschilDialekte, doch wurde der Prozess anch hier dadurch hervorgerufen, dass das $e$ der ersten silbe in unbetonte stollung geriet. Nach Paasonens Auffassung hätte sich dio Vokalreduktion in all den behandelten Fällen wie folgt vollzogr'n: $r>*_{i}>d$, s. a.a.0.97. Die Annahme riner Zwischenstufe *; erscheint jedoch nicht notwendig, ganz zu schweigen davon, dass sich ihre Existenz nicht durch Beloge beweisen lässt.

2.1.3. Paasonen hielt 's für möglich, dass sich bei den hinte!'en Vokalen des Mokscha eine parallele Reduktion $a>*_{u}>\hat{\jmath}$ brweisen liesse, s. a.a.O. Als Beispiele nennt er târa·näa, tuvaráa 'dort entlang', vgl. tona 'jener', tosa 'dort', tot, to 'dorthin';

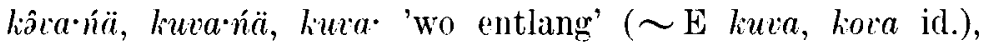
kâna ra, lunarra 'seit welcher Zeit' ( $\sim \mathrm{E}$ dial. konara $)$ vgl. liona 'welcher', losa 'wo', hor, ko 'wohin'. Zu diesen Fällen

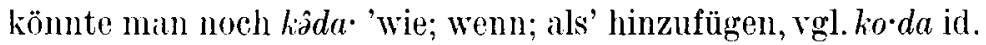

Vielleicht darf nicht ohne weiteres die Erklärung abgelehnt werden, dass sich die Formen mit $\hat{a}$ bzw. $u$ durch Unbetontheit im Satz oder schnelles Sprechtempo aus solchen mit $o$ entwickelt hätten, doch wirkt es bedentend natürlicher, für diese Wörter oinen bereits auf urmordw. Zeit zurückgehenden Vokalwechsel o $\sim u(u$ mundartlich im Mokscha $>\hat{\partial})$ anzunehmen, von dem spaiter in Abschnitt 2.2.3. die Rede sein wird.

2.2.1. Wir stellen also fest, dass es für eine Reduzierung der urmordw. halbengen Vokale der ersten Silbe im Urmordwinischen oder in den hentigen Dialekten sehr wenig Beispiele gibt. Der Wandal $e>$ ist in einigen Wörtern eine Tatsache, doch hat kein cinziger Wandel $o>\hat{d}$ als sicher oder auch nur vermutbar nachgrewiesen werden können. Umso reichlicher sind im Mokscha Belege ron Wörtern vorhanden, wo a und $\hat{a}$ auf die engen Vollvokale $i$ und $u$ zurückgehen. Diese Frage ist in zilhlreichen Untersuchumgen behimdelt worden, s, \%.B. Paaso- 
nen, a.a.O.; D. V. Bubrich $\mathrm{I} z$ istorii mordorskogo vokalizma, Sovetskoje jazykoznanije III $69-91$; Verf., FUF XXIX 295 -296; Gramm. mord. jazykoy 30; O. I. C̆vdaJeva Zapiski mordovskogo naučno-issledoviatel'skogo instituta $18 \mathrm{s.226--}$ 229 ; S.Z. Deva.J Ev Očerki mordovskich dialektov IV 9 und

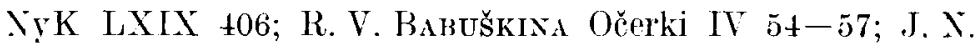
Azrapkix ibill. 257-258; 'T. I. Lomakisa ibid. 305-306. Die in Frage kommenden Reduktionsprozesse sind zu unterschiedlichen Zeiten vor sich gegangen.

2.2.2. Ein sehr alter Wortypus mit reduziertem Vokal ist jener, wo das überall im Mokscha begegnende $\hat{\jmath}$ entstanden ist aus einem vormordw. kurzen $* u$ und $* i$ (sporadisch auch ${ }^{*} e$ ) vor einem akzentuierten $a$ der zweiten silbe. Meistens steht dann zwischen der ersten und zweiten silbe eine mit $r$ beginnende Konsonantenverbindung, z.B. târa. 'Lippe' ( E torva. turva $<$ frühlumordw. *turva $<$ vormordw. *turpa; liârda. 'Mal' $(\sim$ E kirda $)<$ frühurmordw. ${ }^{*}$ kurda $<$ vormordw. ${ }^{*} k e r t a$. Das frühurmordw. kurze $u$ der ersten silbe wurde normalerweise zu o (z.B. tol 'Feuer; Funke' $<<*$ tule; sola 'ungefroren' $<{ }^{*}$ sula), doch begegnete dialektal offenbar schon im Urmordwinischen vor bestimmten Konsonantenverbindungen die Reduktion des unbetonten $u$ zı $\hat{\partial}$. In rinem Wort wie $\mathbf{M}$

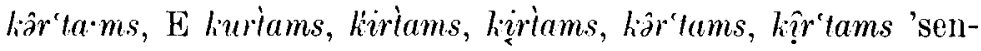
gen, versengen' $<$ frühurmordw. *kurta- (mehr hierher gehörende Beispiele bringen Piasonen, a.a.0. 81-82; C̆udajeva, i.a.0. 227-228; N. F. Cygaxov Mordoyskij gosudarstrennyj Imiversitet: Učenyje zapiski 43 s. $4-5$; М. T. Bıвг ibid. 32 -33) kann die vielgestaltige Vertretung des Erza gänzlich auf rin urmordw. $\hat{\partial}$ zurückgehen. (rinz sicher hat sich aus $\hat{\partial}$ das palatale $i$ einiger Mundarten entwickelt, unklar ist lediglich, ob das $u$ der Variante kuriams ein direktes Erbe des Frühurmordwinischen darstellt oder ob man die Entwicklung frühurmordw. ${ }^{*} u>*^{*}>u$ vorauszusetzen hat. Am unwahrscheinlichsten ist die von Paasonen angenommene Reihe (frühurmordw. $\left.{ }^{*} u>\right) *_{0}>u$, s. a.a.0. $97-98$. Vgl. auch Verf., a.a.O. $333-335$.

2.2.3. Im obigen Abschnitt wurden Wörter behandelt, die in der ersten Silhe in den Ermil-Dialekten eine dreifache Vokal- 
vortretung anfweisen, $" \sim u \sim \hat{3}$. Im Prinzip könnte man eine derartige Vielfältigrkeit auch im Mokscha erwarten, als Folge der bereits im L'mondwinischen infuretretenen Dialektdifforenz'n. Ein Boispiel hierfür kömnten die oben unter 2.1.3. behandeltrn Pronominalstämmo des Mokscha bilden, to $\sim$ tu-〜

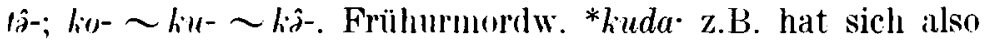
rinerseits - offenbill in unbetonter stellung im Satz - zu lidda entwickeln könnคn, ander'rseits zu ko•da. In der Schriftsprache des Mokscha wir auch in einigen Dialekten haben sich diese Varianten für vrischiedene Bedeutungen spezialisiert: prstere bedeutet 'wenn', letzterr 'wie', s. Potapkix-Iy.Jar ekov Mokšanskô-russkij slovaŕ 107, 117; Derajer, Očerki II 264; Lomakina, a.a.0. 291.

2.2.t. Ein indirektes Zeugnis für das Alter der Vokalreduktion bieten U.a. dir Wörtor E lišna. E M šna 'Riemen' und E II skal 'Kuh'. in denen der unbetonte Vokal der ersten Silbe bereits im Urmordwinischen geschwunden ist. Ersteres Wort hatte die frühurmordw. Iantgestalt *šukšna $(<$ balt. šikšna)

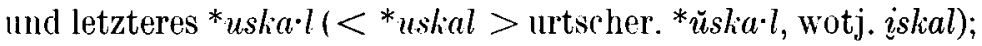
ihre Entwicklung zu Einsilbern lässt sich schwerlich anders vorstellen als durch die Vermittlung der urmordw. Formen *.âališna,${ }^{*} \hat{a} s k a \cdot l$. Um eine späte mokschanische Parallelerscheinung dieses Schwundes handelt es sich dann, wenn die Formen des Schrift-Mokscha rom 'Typ mрва 'Lippe', нрда 'Mal' eine Aussprache wiedergeben. die wenigstens für einige Mundarten gilt. Generell geben die mordwinischen Forscher jedoch zu verstehen, dass der Vokal der ersten Silbe im Tỵus târra, kârda ausgesprochen wird, auch wenn er in der normalen Orthographie nicht in Erscheinung tritt. S. besonders I. (r. Tultajer Mordorskij gosudarstrennyj prdagogičeskij institut: Sbornik studenčeskich naučnỵch labot $1 \leqslant i t-i 6$.

2.2.5. Es gibt nun ein Reih? von Wörtern, wo dem mokschanischen â überall im Erza ein " entspricht. Hierher gehört z.B. II pôl'ta'ms, E puliams 'brennen (trans.), verbrennen'. nach den uns zur Verfügung stehenden Angaben fermer $\mathbf{M}$ mârda.ms (in der Schriftsprache . $p \partial a . u c)$ ), E murdams 'umkehren, zurückkehren' Ind II kar't'ś • ( $\hat{\partial}>\partial$ vor mouilliertem Konsonant), E kurt'ś 'schultrijoch (zum Wassertragen)'. In 
der ersten Silbe dieser Wörter steht ein späturnordw. $u$, dils normalerweise zurückgreht auf vormor $d w .{ }^{*} o,{ }^{*} \bar{o}$ oder ${ }^{*} \bar{u}$ und etymologisch also andorer Herkunft ist als das in 2.2.2. und 2.2.3. behandelte frühmmordw. ${ }^{*} u$. Jedoch kilnn aluch der letztgenannte Vokal, der einen vormordw. kurzen, engen ladut vertritt, ausnahmsweise erhalten sein sowohl im Erzal als auch in den meisten Mokscha-Dialekten. Schon unter Punkt 2.1.3.wurde als Beispiel für einen solchen Fall E liwva. M liwva 'wo entlang' angeführt. Ein ähnliches Wort liegt ror' in E M liundams 'anfassen, ergreifen; fangen; unternehmen, anfangen' ( < vormordw. *kunta- $>$ fi. kunti- [ $j$-Ableitung| 'pflücken', lp. god'de- 'töten; [Fische] fangen'). Zuweilen wäre aluch - u.a. gerade beim Wort kundams - die Entwicklung frühurmordw. ${ }^{*} u>*_{0}>u$ denkbar, wobei dann der Wandel von o zu $u$ rine auf den schliessenden oder dehnenden Einfluss der nachfolgenden Konsonantenverbindung zurückgehende Folgeerscheinung wäre, vgl. Verf. a.a.O. 329, 334. Das späturmordw. $u$ - ob nun anzusehen als Ergebnis regelmässiger oder sporadischer Entwicklung ist im Erza erhalten, im Mokscha jedoch sehr oft zu â reduziert.

2.2.6. Typisch für die Verhältnisse im Mokscha ist, dass sich das späturmordw. $u$, während es einerseits $z u \hat{\partial}$ wurde, andererseits in einigen anderen Formen der diesburgüglichen Wörter erhalten hat, wodurch der paradigmatische Wechsel $u \sim \hat{a}$ zustande kam. Der Wechsel zwischen diesen Vokalen kann auch ils recht unzusammenhängende, interdialoktale Erscheinung vorkommen. (Das Mokscha besitzt zumindest in mehreren Dialekten $u$-Wörter, in denen der Wandol $u>\hat{z}-$ soweit bekannt - überhaupt nicht auftritt, doch sind sie in diesem Zusammenhang für uns nicht interessant.)

Nach Paasonen bestehen gewisse Nuancenunterschiede in der Reduktion von $u$ and auch $i$. Diese Vokale sind allgemein "etwas schlaffer» altikuliort, wenn der Druckalkzent von ihnen auf die nichtersten Silben übergogangen ist. Der Prolativ von kud 'Haus' z.B., kudga, wird im Dialekt von Pšenevo fast wie liadga ausgesprochen. Ein ähnliches schwach reduziertes $\partial$ findet sich in den Formen $k \hat{\partial} l a \cdot n$ 'ich sterbo' und kiâlâma 'man muss sterben', während die Formen kulâms 'sterben', kulị 'er stirbt' ein $u$ haben. Ein eindeutig reduziertes â begegnet vor allem 
in Wörtern, deren erste Silbe in keiner Form akzentuiert ist, z.B. Pšenevo ârda·s 'Kot'. Besonders die Mundart von Selišče wird durch die Erscheinung charakterisiert, dass sich das aus $u$ in unbetonter Stellung entwickelnde $\hat{\partial}$ auch in Formen durchsetzen konnte, die den Druckakzent auf der ersten Silbe haben, wodurch ein Wechsel ausgelöst wurde wie putį $\sim$ pâtį 'er setzt' (vgl. pâta‘n 'ich setze'), mukâr 〜 mâkâr 'After' (s. a.a.O. 97). Cudajeva erwähnt, dass im Dialekt von Staroje P̌̌enevo vor allem das unbetonte $u$ zu $\hat{\partial}$ geworden sei, z.B. ôrma 'Krankheit' ( dial. und schriftsprachl. urma $)$, kâdri ( dial. und schriftsprachl. kudń $\varepsilon^{\circ}$ ); die Erscheinung begegnet jedoch nicht in allen Wörtern, z.B. pulaj 'Teil der Frauentracht', s. Očerki III 30. Devajev notiert den Wandel des unbetonten $u \mathrm{zu} \hat{\partial}$ als regelmässig in den Südostdialekten des Mokscha; dieselbe Erscheinung würde - wenn auch nicht regelmässig - in den Südwestdialekten bekannt sein, s. Očerki IV 9, NyK LXIX 406. Aus dem Dialekt von Temjaševo hat Babuškina z.B. âdâma . 'Schlafen, Schlaf' ( dial. und schriftsprachl. udâma.) und kânda·ms 'erfassen' ( dial. und schriftsprachl. kunda $m s$ ) aufgezeichnet, s. a.a.O. 55-56. Zahlreiche Belege sind ferner angeführt worden aus dem Dialekt von Kolopino, u.a. pâpams 'stechen, beissen' ( dial. und schriftsprachl. pupa·ms), kârat' 'lange' ( dial. und schriftsprachl. $\left.k u v a \cdot t^{\prime}\right)$. An dem Wandel $u>\hat{\partial}$ konnten in diesem Dialekt auch die jungen russischen Lelınwörter teilnehmen, z.B. trâpka 'Pfeife' (schriftsprachl. trubbka, Druckakzent also auf der ersten Silbe) < russ. mṕ $\dot{y} \sigma_{k} a$. Der Wandel ist jedoch nicht konsequent durchgeführt; das akzentlose $u$ hat sich auch erhalten können, z.B. nuram 'Wiege', kutška 'Mitte, Mittelpunkt', kurka 'Truthahn' < tat. kurka. Andererseits hat auch ein akzentuiertes $u$ zu â werden können, z.B. pârâm 'Bremse' ( dial. und schriftsprachl. pu•râm). S. Azrapkin, a.a.O. Im Dialekt von RybkinoMamolajevo begegnet der Wandel $u>\hat{a}$ sowohl in der akzentlosen als auch in der akzentuierten Silbe, wenn auch nicht konsequent, z.B. śma ( $\hat{\partial}>$ o nach einem mouillierten Sibilanten) 'Trog' ( dial. und schriftsprachl. śuma $)$, pâpârda·ms

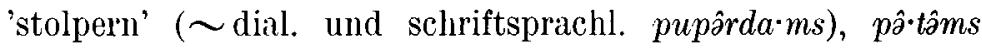
'setzen, legen, stellen' ( dial. und schriftsprachl. pu•tâms). 
Lipatov, von dem diese Angaben stammen, zählt noch eine ganz Reihe anderer Dialekte mit ähnlicher Vertretung auf, s. a.a.(). 181.

2.3.1. Wir kommen nun zu den Fällen, wo der reduzierte Vokal im Mokscha zurückgeht auf einen urmordw. vorderen Vokal. Es ist wahrscheinlich, dass das rormordw. * $\ddot{a}$ der zweiten Silbe in der frühesten Phase der Entwicklung des Mordwinischen unter denselben Bedingungen akzentuiert war wie der entsprechende lintere Vokal $a$, nämlich nach einem kurzen, engen Vokal der ersten Silbe, vgl. Verf. a.a.O. 298-299. Somit ist es theoretisch möglich, dass der Vertreter des vormordw. $*_{i}$ oder $*_{\ddot{u}}$ der ersten Silbe in unbetonter Stellung frühzeitig zII *a reduziert werden und weiterhin völlig wegfallen konnte, eine Parallelerscheinung zu dem unter 2.2.2. und 2.2.4. behandelten Wandel vormordw. ${ }^{*} i,{ }^{*} u>$ frühurmordw. ${ }^{*} u>$ dial. $* \hat{z}(>\varnothing)$. Doch dürfte es keine sicheren Belege geben für Wörter mit erhaltener Silbenzihl, die in der ersten silbe den Vertreter eines so entstandenen urmordw. *a hätten. Da in den meisten Erza-Dialekten keine reduzierten Vokale begegnen, wäre *a dort jedenfalls zu einem Vollvokal geworden, in erster Linie wohl zu $i$. Es ist jedoch anzumerken, dass auch in den Er\%a-Dialekten, die in der ersten Silbe reduzierte Vokale aufweisen, letzter' ziemlich ausnahmslos Entsprechungen des auf einen urmordw. hinteren Vokal zurückgehenden à zu sein scheinen. Aus dem Dialekt Kažlytka hat Verf. zwar bei Paasonen (a.a.(). 54, 98) zwei Wörter gefunden, in denen ein reduzierter Vokal auch im Mokscha einem auf ninen urmordw. Vordervokal zurückgehenden a entspricht: p!šlia.dums 'ïussern' ( E pišskadoms, pškadems, II paškä̉dams); m?źa·ra 'wieviel'. Das letztere Wort kim brreits unter Punkt 2.1.2. zur sprache; wie in dem Zusammenhang festgestellt wurde, kann das M a, E ? dieses Wortes nicht ummittelbar anf einen vormordw. engen Vokal zurüchgehen, sondern es entstand im Resultat der Entwicklungsieihe vormordw. ${ }^{*} i>\operatorname{urmordw} e>>$ a. In Wort puška dums diagegen wird es sich um einen reduziejten Vokal handeln können, der schon aus der älteren urmordw. Zeit stammt.

2.3.2. Die Frage nach einer frühen Reduktion des vormordw. engen Vordervokals ergibt sich besonders anhand solcher ein- 


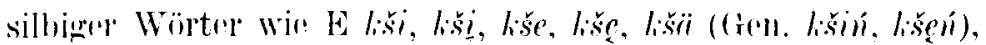

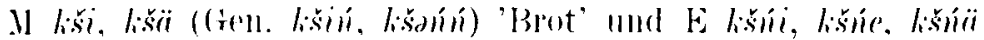

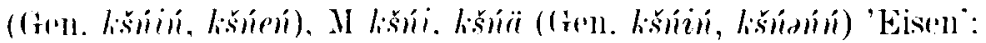

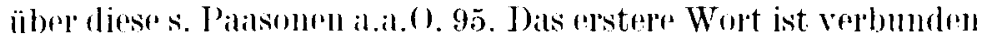
worden mit fi. kygrsä 'Brot, Iaib'. das letztere mit tscher. kürrnü̈ 'Eisen'. Falls die Etỵmolorien stimmen, kamn für diese Würter folgende Entwickhngr angremommen werden: *liärsä $>$ *liarzii

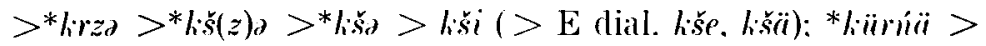

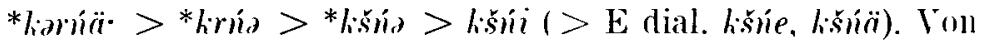
de'm Wort für 'Ejsen' arscheint in rinigen Erza-D)ialekten auch die Form lišine (s. F. .J. Wrenemaxx Grammatik der Ersamordwinischen Sprache 128. I). T. N.1IKIx Voprosy mordovskogo jalykoznanija |1969| S. 73), deren $i$ in der ersten Silbe in Lichtr obiger Erklärmug rin sokundärer. nichtetỵologischer l,allt wäre.

Sowohl im Mokschal als allch im Erza begegnen ausserdem zienlich viel Wörter, boi denen der Vertreter des urmordw. Vordervokials in der arsten Silbe dialektwoise geschwunden ist. Hierher gehören 1 .il. E M lišrian E hešnan 'ich niese'; E p̈ra, II prí $\sim$ folkl. E pira. M jire. 'Kopf, Hampt; Gipfel; Wipfel;

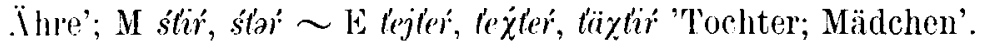
All diese Fille, deren sich mehr bei Palasonen a.a.O. 52-56 und Bulbrich, Istoričoskajja grammatikiz erzjanskogo jazyka 11, 36-38 finden, müssten gesondrrt untersucht werden, wenn man Klarhcit darüber haben will, ob möglicherweise ein reduzierter Vokill als Zwischenstufe brim schwund des Vokals der ersten Silbe gredient hat. Zweiffllos haben die Schwundfälle ein verschiedenes Alter und rertroten prinzipiell mehrere Typen; in diesem Zusammenhang sind sie nicht sonderlich wichtig.

2.3.3. Generell ist zu sagen, dass sich im Auftreten des palitalen reduzierten Vokils des Mordwinischen nicht solchr schichten unterscheiden lasson wie beim $\hat{\partial}$. Die einzige (iruppe von d-Fällen mit eindentiger Entsprechungr beim Vordervokal sind dir einen Wechsel $u \sim j$ antweisenden Wörter im Mokscha. die unter 2.2.6. behandelt worden. Wir untersuchen mun den rntsprechenden rordervokalischen Wechsel im Uokscha, $i \sim$ д. Zugleich sei noch an dir berits in Abschnitt 1. besprocheni Erscheinung erinnert, disss sich dip Lanute a und a im grugen- 
wärtigen Mokscha vielerorts vermischt haben. Es können sogar auch Wechselfälle auftreten wie $u \sim \Rightarrow$ und $i \sim \hat{\partial}$. Interdialektalen $\partial \sim \hat{\partial}$-Wechsel weist I.a. Pšsenevo krála $n \sim$ Selišče kấrda・n 'ich halte' ( kiŕdams 'halten') auf. Es ist nach Paasonen eine normale Erscheinung, dass das unbetonte $i$ der ersten Silbe entweder immer etwas schlaffer artikuliert wird als das betonte oder dann mehr oder minder reduziert ist, s. a.a.O. 97. Nach Devajev herrscht in den Zentraldialekten des Mokschil sowohl in betonter als auch in unbetonter Stellung i.a. $i(k i \cdot r-$ lams, kiŕl(a.n); im Südostdialekt ist der Wechsel des akzentuierten $i$ mit dem nichtakzentuierten a $\left(k i \cdot \cdot^{\prime} d a m s \sim k a r^{\prime} d a \cdot n\right)$ regelmässig; den Südwestdialekten spricht der Verfasser eine derartige Regelmässigkeit ab, "häufig erscheint jedoch das Phonem (a) auch akzentuiert" (ka'ŕdams, kard 'a・n), s. Očerki IV 9. (Das z der bei Devajev in russischen Buchstaben geschriebenen Beispiele ist oben durch $ə$ transliteriert; in der ungarischen Fassung des in Frage stehenden Aufsatzes in NyK LXIX ist das $z$ des Originals durch $\ddot{i}$ ersetzt.)

3.1. Wenn wir nun zur Behandlung der reduzierten Vokale der n i c h t e r's t e n s i l b e n übergehen, darf zunächst nicht vergessen werden, dass dor Unterschied zwischen a und $\partial$ in diesen Positionen letztlich anf die rormordw. Vokalharmonie zurückgeht. Niemand bezweifelt die Existenz einer vormordw. $\ddot{a}$ : a -Harmonie. a ist der normale Vertreter von ${ }^{*} \ddot{a}$ und â von *a, z.B. M selma- 'Auge' <* silmä; parâ- 'gut' < *para. Seinerzeit wurde allgemein ingenommen, dass es neben der Harmonie $a$ : $a$ auch eine $e: e$ gegaben habe. Hierauf würde auf den ersten Blick der Tatbestand hinweisen, dass ein $*_{e}$ nach einem Vordervokal vertreten ist durch $\partial$, nach einem hinteren Vokal jedoch allgemein durch $\hat{\delta}$, z.B. veñoš 'Kahn',

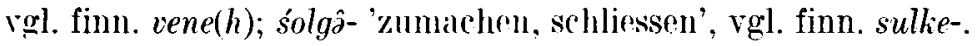

Doch finden sich im Mordwinischen stichhaltige Beweise für die Auffassung, dass auch nach einem hinteren Vokal das vormordw. ${ }^{*} e$, wie im heutigen Finnisch, palatal war. Verf. hat diese Frage bereits in den FUF XXIX 298-299 kurz behandelt und kann nun seine damalige Darstellung vervollständigen. Wie bekannt qribt es eine ganze Reihe alter e-Stämme, wo nach ainem hinteren Vokal das urmordw., im Mokscha noch heute 
rhaltent a begronnt: hol'ga- 'triefen usw: <*kulke-; kul'a'hören' < *läle-; mol's- 'gehen, hingrohen, hinkommen' <*mule-

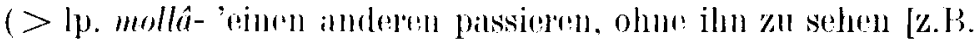
im Waldel'); pana- 'backen; trubon, vertreiben' < * pane-; pora'katuen' < * pure-; suftom 'Sieb' $(\sim \mathrm{E}$ sucteme, sufterie $)<{ }^{*}$ suftoma, Ableitung von * sufic- 'sieben' (vgl. Paasonen, MSF()u XLI

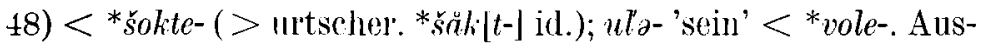
ser diesen bereits bekinnten Beispielen hier noch zwei nene: sańd'ə- 'roden, lichten (den Wald); durch striegeln reinigen' < *sante- (> lps, U, L, N suod'dâ-'entästen, lichten (den Wald); (ab)schneiden (Schuhheu, Reisig, Stücke rom Fisch usw.); schnell abhäuten; mit dem Messer kastrieren'; šud'ə- 'fliessen, rimen' $<{ }^{*} s \bar{o} \delta e-$ bzw. ${ }^{*}-\delta$ '- $(>\operatorname{lpP}, \mathrm{N}, \mathrm{I}$ suoddâ-'durchdringen, durch ein Loch o. dgl. [zischend] strömen [von der Luft]'). Im Lichte dieser Fälle ist es natürlich, dass man allch z.B. bei den Wörtern kolmồ- 'drei' <*kolme; potšlià- 'Rohr, hohler

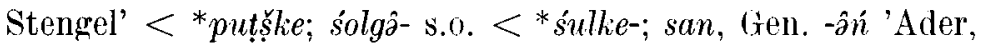
Sehne' < *sōne; tol, Gen. -àn 'Feuer' < *tule 'Funke' auszugehen hat von den frühurmordw. Formen *liolma-, *potšlia-, *solga-, *san, -an, *tol, -an.

3.2.1. Die heutigen Verhältnisse im Mordwinischen erweisen sich als Resultate zweier, im Urmordwinischen wirksamer, gleichsam entgegengesetzter Tendenzen.

3.2.2. Einmal herrschte das Streben, dic Vokalharmonie zu vervollständigen, indem das einem hinteren Vokal folgende a mit dem aus einem unbetonten $* a$ ontwickelten $\hat{\partial}$ verschmolz $\left({ }^{*} k o l m a>{ }^{*} k o l m a \hat{~ u s w .) . ~ D i e s e r ~ W a n d e l ~ w u r d e ~ v e r h i n d e r t, w e n n ~}\right.$ dem a ein $j$ oder ein mouillierter Konsonant oder eine Konsonantenverbindung voranging, deren eine Komponente ciner der' genannten Laute war. Dies zeigen die Wortstämme uja- 'schwimmen' <*uje- bzw. *oje-; *oj, Gen. -an (> E oj, M $v a j)$ 'Fett, Butter, Öl' <*voje; laks'a- 'behauen, abbeilen' < *lakise- $(>$ lp.

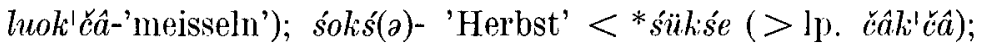
muśka- 'waschen' < *muśke- (> tscher. mǔš $[k-1)$ bzw. *mośke(> estn. mõske-).

Der Ausgleich fülırte dazu, dass andererseits dor auf *a zurückgehende und also velare reduzierte Vokal mit dem nach $j$ oder nach einem mouillierten Konsonanten stehenden a zu- 
sammenficl. Dabei trat also die dem obigen Wandel $\partial>\hat{\partial}$ entgegengesetzte Entwicklung $\hat{\partial}>$ a ein. Z.B. muja- 'finden' $<$ ${ }^{*}$ mujà- $<{ }^{*}$ moja- (vgl. karel.-olon. muja-, dessen Entsprechungr in ler sïdlichen Gruppe der osfi. Sprachen auf ein urspr. o hinw'ist. s. SKES 349); kut'śa- 'klettern, hinaufsteigen' <*kut'śá$<{ }^{*}$ liot'su- (> tscher. kuzâ-); mar, Gen. -дй Apfel; (in Zusammenset\%ungen) Beere' $<{ }^{*}$ mar, - дn $<{ }^{*}$ marjo $<{ }^{*}$ marja; uŕd'Sklave; Lohndifner' < *urâ bzw. *urja < *urjâ< < orja; uzar

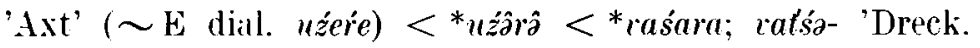

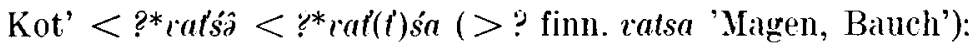

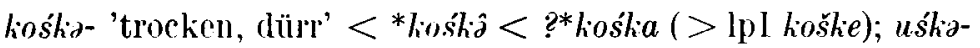
'Draht' <*uśliâ < ?*roślia (> lp. *eeślä > I vešli 'Kupfer').

In einigen Fällen kann man nicht wissen, ob das einem $j$ oder mouillierten Konsonanten folgende a der Vertreter eines vormordw. * ${ }^{*}$ oder * $a$ ist. Z. B. kaź 'schenken, beschenken' <

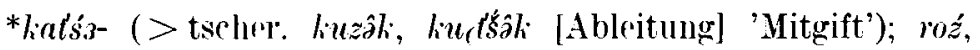

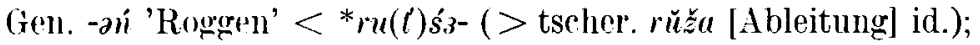
kajgə- 'hell lanten, klingen' <*liajkiz- (> finn. kaiku [Ableitung] 'Widerhall, Echo'); rajma- 'Atem, Atemzug, lebendes Wesen' < * vajms (> finn. vaimo 'Weib', estn. vaim, $-u[\mathrm{Ab}-$ leitung] 'Geist, Seele'); das mit dem letztgenannten mordwinischen Wort zusammengehörende Verb rajma- 'sich beruhigen, ruhen' weist auf einen ursprünglichen *a-Stamm hin, der Auslautvokal des in Frage stehenden stammes kann jedoch auch inalog sein.

Mit Ausnahme ganz jungrer Lehnwörter hat ras Mordwinische nur wenig Wörter, in denen nach den senannten Konsonanten ein hinterer Vokal auftritt: M aigàr, E ajgor 'Hengst' (< tat. ajyâr; dieses Wort wurde offenbar so spät ins Mordwinische rntlehnt, dass das in Limordwinischen wirksame Lautgesetz nicht mehr galt); E końor $(<$ *lioniâs) 'Papier' (auch dies ein Lehnwort; s. zuletzt A magyar nyelv törtíneti-etimológiai szótára II 614); U ṕizâl, E pizóol 'Vogelbeere; Vogelbeerbaum' (handelt es sich hier um einen späten Wandel $*_{z}>z$ ? ); E $k$ uctol 'Schote' (offenbar sekundär mouilliert, vơl. M kuftâl id.).

3.2.3. Die zweite, durch die hentige Vertretung reflektierte urmordwinische Erscheinung war die, lass der Stammtypus laźa-, ứa- mit mouilliertem Stammkonsmulutismus in Stäm- 
men vom Typ *kolga-, *solga- mit der Vokalharmonie-Tendenz zu konkurrieren begann. Wo die Tendenz zur Vokalharmonie den Sieg davon trug, vollzog sich der Wandel a $>\hat{a}$ und der Stammkonsonantismus blieb unmouilliert erhalten (z.B. *solga- > śolgâ-). Gewamm jedoch die Tendenz zur Mouillierung die Oberhand, blieb das z palatal erhalten, während der Stammkonsonantismus mouilliert wurde (z.B. ${ }^{*} k o l g \partial^{-}>$kol'ga-). Eine Regelmässigkeit lässt sich in dieser Zweiteilung nicht beobachten. Auffallend ist jedoch, dass es sich bei allen bisher genannten Wörtern mit sekundärer Mouillier'ung (kol'ga-, kul'ə-, molə-, pańə-,

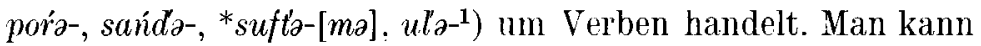
deshalb fragen, ob sich die Moullierung zuerst in den Verbalformen vollzogen hat, die in der zweiten Silbe ein $i$ haben (z.B. pori 'er kiult'), wonach sie sich dann in gesimten Paradigma durchgesetzt hätte. Die Nominalflexion weist ja keine Formen mit einem $i$ in der zweiten silbe auf. Die Frage mag offen bleiben; zu vermuten ist allerdings, dass die Antwort negativ ausfallen wird. Das ist ganz sicher der Fall, falls die Etymologie mordw. kar (Gen. -əń) 'Bastschuh' finn. kuori 'Rinde' $\left(<{ }^{*}\right.$ kòre $)$ zutrifft. Es gibt noch einige andere Substantive, deren Etymologie unbekannt ist, die denselben Typ vertreten wie $k a \dot{r}$ und die vielleicht ebenso zu erklären sind.

3.2.4. Wir brauchen uns hier nicht mit der im Urmordwinischen entstandenen sekundären Konsonantenmouillierung im Ganzen zu befassen. Auch hinsichtlich der vordervokalischen Wörter vom Typ śl’mə-, ieñaš könnte man zwar erörtern, ob der allgemeine Palatalisierungsprozess in Wörtern seinen Anfang $11 \mathrm{hm}$, die einen vormordw. mouillierten Konsonanten aufwiesen (z.B. sed' 'Kohle' < *'ï $\delta^{\prime}$; k'enar' 'Vorderarm, Elle' $<{ }^{*}$ kïnier; sirńa- < urmordw. *sirna 'Gold' < *sernä) oder einen, aus einer auf $j$ endenden Konsonantenverbindung entwickelten frühurmordw. mouillierten Konsonanten (z.B. *kela 'Breite' <*kiljä bzw. *küljä 「>lp. gâl'lje 'weit, geräumig, breit']; nil'a- 'vier' < ${ }^{*}$ neljä bzw. *ń-; pilla- Ohr' < *peljä). Wir'

1 Im Worte šuda- 'fliessen, rinnen' kann die Mouillierung in dem Falle vormordw. Herkunft sein, wenn das Wort auf die Urform ${ }^{*}{ }^{*} \bar{o} \delta \delta^{\prime} e-\mathrm{zu}$ rückgeht und die Entwicklıng ${ }^{*} \delta^{\prime}>a^{\prime}$ und nicht ${ }^{*} \delta^{\prime}>{ }^{*} d>d^{\prime \prime}$ durchgemacht hat. 
beachten jetzt nur den Limstand, dass im Iokscha dio einem reduzierten a vorangehenden Labial- und Palatalkonsonanten allgemein nicht monilliert sind. während im Er\%al in de'n entsprechenden Formen, wo a sich zu rinem Vollvokal entwickelt hat, die erwähnten Konsonante'n monilliert sind, z. B. V seram. $\sim$ E sqciems 'verzhren. anfessen, -fressen', $\mathrm{M}$ simam.s $\sim \mathrm{E}$ simems. 'trinken', II liolgams $\sim$ E lol'gems 'triefen, rimmen'. Dir: oben in Punkt 1. zitierte Regel von Palsonen ist im Lichte seiner Mokscha-Texte nicht ramz ohne Ansmahmem. bogegnem doch dort mitunter der Labialkonsonant und recht häufior dor Palatalkonsonant nouilliert vor einem z, z.B. siäpania 'Galle' Dem., säicaź 'sie nithmen es', piilgań 'Fuss' Gen., pelian' 'Bauch' Gen. Andererseits findet man instelle ron a auch fin $g$ brzoichnet (wie in der hrutiqen mordwinischen schuiftsprache), woror der Konsonant natürlieh nicht monilliert ist, z.B. setmânza 'soinr Aựen', peliâšlan so gross wie der Bauch'. I î Frage, wir dieso verschiedenen Brezeichmungswrisen zu bruptrilen sind, magr offen bleiben.

Paasonen vertmat die Meinmo, die Labiale und Palatalr seien im Crmordwinischen rol' a monilliert gewesen, wie z.B. spinf' urmordw. Rekonstruktionen rämb̈̈̈- 'Warm', koskïz- 'trocken' und lišniz- 'Pferd' zeigen, s. a.a.(). 105. 107. An diesen' Auslegung wird man kaum etwas auszusetzen haben. Palasonens Inschaung wird schon durch die T'atsache als richtig bewiesen. dass im Mokscha der Stammkonsonant vor einem allf o zurückgehenden $\varepsilon$ im Wortanslaut monilliert ist, wir in don Er"zaEntsprechungen der diesbezüglichen Formen (z.B. II sef ine.

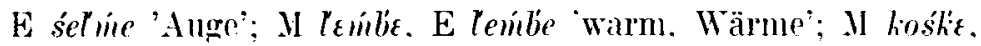
E hoske 'dürr'). In Vokscha ist die urspriunglichr Palartaiität des Vertreters von a im Wortiuslaut erhalten gebliebrn. während im inlantenden a ein Streben nach Velarisicrungr zum Ausdruck kam. Dialektwrise diürte o sogar völlig zusammengefallen sein mit $\hat{\partial}$, was jordoch nur nach Labialen und Palatalen eintrat. nicht nach Dentalen. Dass sich $ə$ in der letztgenamnten Stellung rordervokalisch mhalten hat, beruht wiederum damauf. dass die ihm vorangehenden Dentale $d, t, L^{\prime}, i, r^{\prime}, R^{\prime} . s^{\prime}(\dot{s}), t$,

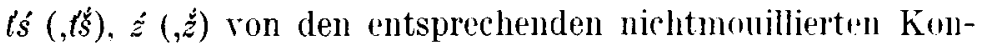
sonanten getrennte Phoneme sind, welcher. ['ntersehierl ryalten 
bleiben sollte, weshalb auch keine Velarisierung des auf diese mouillierten Konsonanten folgenden a in Frige kam. Die Labiale und Palatale dagegen kennen keine phonologische Palitalitätskorrelation, so dass die Velarisierungstendenz des a nach diesen Konsonanten verwirklicht werden konnte; in dem Zusammenhang vollzog sich automatisch eime Entmouillierun!r der mouillierten Allophone der Labiale und Palatalle, z.K.

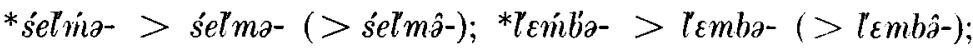
*kośka- > kośka- (>kośkî-).

Es gibt jedoch noch andere Beweise für eine frühe Mouillierung der Palatale vor a. In dieser Stellung ist das interrokilische Einzel-* $k$ in vordervokalischen Wörtern im Umordwinischen regelmässig zu $j$ geworden, z.B. mijz- 'verkaufen' < ${ }^{*}$ mèke-; neja- 'sehen, erblicken' < *näke-; *'eja- (> E t'ejeII tija-) 'machen, tun' <*teke-. In einigen Fällen ist auch ein nach einem Hintervokial stehendes ${ }^{*} k$ vor a zu $j$ geworden: saja- (>M saja- 'kommen, ankommen', E saje- 'nehmen, wegnehmen, sich bemächtigen; bekommen, erhalten') <*salie-; tuja- (> M tuja-, E tuje-) 'bringen, holen' <*tōke-. Die Entwicklung ging so vor sich: ${ }^{*} k>{ }^{*} \gamma>{ }^{*} \gamma^{\prime}$ (Mouillierung des Palatals also) $>j$. (Es ist möglich, dass sich das ${ }^{*} k$ der fiu. Stämme ${ }^{*}$ méke- und *tōke- nach einem langen Vokal bercits im Vormordwinischen zu $*_{\gamma}$ entwickelt hatte.) In den letztgonannten Wörtern aber, die in der ersten silbe einen hinteren Vokal haben, scheint die Tendenz zur Palatalisierung mit der zur Vokalharmonie konkurriert zu haben. Infolge der ersteren Tendenz entwickelte sich der Stamm *tuy ${ }^{-}$in den frühurmordw. Dialekten durch Vermittlung der Form *uýa- zur Lautgestalt tuja-, durch den Einfluss der letzteren Tendenz jedoch zu *tuvô- (unter der Voraussetzung, diss das von Aug. Alilqvist für das Erza angeführte tuvoms faktisch besteht). Auch das eigenartige ścva- 'nehmen' im Mokscha, kaum zu trennen rom Erza-Wort saje-, kann darauf hinweisen, dass alls der ursprünglichen Form *sake- auch die auf der Tendenz zur Vokalharmonie beruhende Variante * savâ- entstanden ist, woraus im Mokscha durch einen exzeptionellen Wandel ${ }^{*} a>\varepsilon$ dic heutige Form zustande kam. Die an sich interessante Entwicklung ron fiı. ${ }^{*}$ in IIordwinischen kamm in diesem Zı- 
sammenhang kluz gestreift werden. Das im Erza sporadisch begegnende $e \eta^{\prime}$ 'Eis' $<{ }^{*}$ jäye hat die aus * ${ }_{\eta}$ in den rordervokalischen Wörtern des Urmordw. vor $*_{\partial}$ entstandene novillierte. Variante bewahrt. Möglicherweise geht anf dieses $\eta \dot{n}$ sowohl das $j$ (z.B. M $j \varepsilon j, \varepsilon j$, E $e j, i j$ 'Eis'; M šejor, E tšejer 'Malus' < frühurmordw. * šérar) wie anch das dialektal anstelle von $j$ auftretende $i(r)$ zurück (z.B. E er 'Eis', tšeier' 'Maus'). PaAvo Ravila hat dirauf hingewiesen, dass in der Entwicklung des ${ }^{*} l_{i}\left(*_{y}\right)$ und $*_{y}$ mordwinischerseits von Anfang an ein bestimmtes Schwanken hat herrsehen könne'n. s. MSFOu LXVII 359.

3.2.5. Die reduzierten Vokale haben mit dem nachfolgenden $j$ bereits im Umordwiniwhen versehmolzen! Vokale gebildet. Aus der Verbindung a $+j$ rntstand $i$, z.B. ${ }^{*}$ nil $a-j>n i l i 3$. Ser.Prs. 'er verschlingt'. Dif Verbindung $\hat{z}+j$ entwickelte sich in zwei verschiedenen Wrisen. Steht ror dem $\hat{\partial}$ ein Dentalkonsonant, kam es zur Entwicklung $\hat{\jmath}+j>i$ (im Erza hat sich auch urmordw. $a+j$, in Mokschal erhalten. zu $i$ entwickelt). Wenn ror dem a jedoch ein Labial oder Palatal steht. kam es zu dem Wandel $\hat{a}+j>i$. Wie oben angeführt, ist der einem urmordw. $\hat{\partial}$ vorangehende Konsonant bzw. die Konsonantenverbindung immer ummouilliert. Die Jentalkonsonanten vor $i(<* \hat{a} j)$ haben sich auch als solchr erhalten. z.B. ${ }^{*} k$ and $\hat{\partial}-j>$ kandi 'er bringt'. *kulà-j > kuli 'er stirbt' (urmordw. und II pala-j 'er küsst' $>$ E pali $)$. Im Zusammenhang mit der Entwicklung $\hat{\partial}+j>i$ dagegen sind die Labiale und Palatale vor $i$ mouilliert, z.B. *jacà-j > jå̛ $i$ 'er teilt'; *solgâ- $j$ $>$ solgi $i$ 'er verschliesst'; *usk $\hat{\partial}-j>$ uski 'er fülırt' ( ${ }^{*} k a r m a-j>$ E liarmi 'er beginnt'; *lapa-j > E laṕi 'er klatscht'; *kurva-j $>$ E kurvi 'es lodert'). Die zweifache Entwicklung der Verbindung $\hat{\partial}+j$ ist offenbar so zu verstehen, dass infolge der starken Assimilationswirkung des palatalen $j$ das Ergebnis stets $i$ gewesen wäre, falls der vorangehende Konsonantismus diese Entwicklung nicht in bestimmten Fällen verhindert hätte. Es geht darum, dass falls auch nach Dentalen $\hat{\partial}+j$ zu $i$ geworden wäre, die Dentale unumgänglicherweise palatalisiert worden (z.B. *kańdi, *kuli pro kandị, kulị), m.a.W. zu anderen, mouillierten Phonemen geworden wären; weiterhin wären die diesbezüglichen Wörter in einen anderen Stammtypus (z.R. *kańda-, 


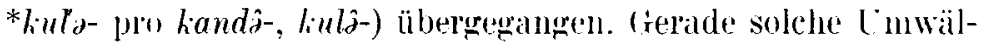
zungen wollte man rerhindern, weshalb die Entwicklungr ron ${ }^{*} \hat{j}+j$ nach Dentalen auf der $i-S t u f e$ stehen blieb. Xach Latbialen mod Palatalen komnte der Wandel $\hat{z}+j>i$ puhig rintreten. dia rir rlurch $i$ remursaldehten Monillierungsprozesse $g=\dot{y}$. $k>k, m>m, p>\dot{r}, r>i$ keine phomogrische Bedrutun hatten.

3.2.6. Es ist selbstrerständlich, disss die nicht an dor Palatalitaitskorrelation betriligrten Iabiale und Palatale nicht allein als Stammkonsonanten zwischen hinteren Vokalen und einem palattalen a auftreten kömmen, in Wörtern also vom Typ kul'a-. pora-. Wenn es nämlich neben z.B. den urmordw. Stämmen šam

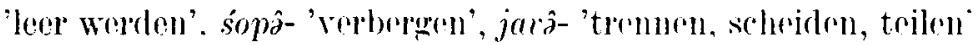

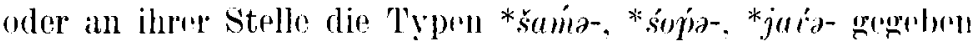
hätte, damn wären dir mouilliorten labiale und Palatale selhständige, monillierte Ihomeme gewesen. solche hab'n sich abry - wie gesagt - alls dem rinen oder anderen frund im Mordwinischen nie entwickelt. '/wischen einem hinteren Vokal unil a konnten die Labiale und Palatale lediglich in Konsonantenverbindungen stehen, deren eine Komponente $j$ war (z.B. *ajno 'Geist, Seele'; *kajgo- 'hell lauten, hlingen') oder ein mouillierte's Dentalphonem (z.B. *sufta-mo 'Sieb'; *laks'a- 'behauen, abhaven'; *muskio- 'waschen'), und grerade diese anderen Konsonanten bestimmten den palatalisierten Charakter der Konsonantenverbindungen; die Iabiale und Palatale aber, die monilliert oder nichtmouilliert sein konnten, hatten in dieser Hinsicht keine Bedeutung. Aufschlussreich ist die Beobachtung. dass das aus der Verbindung $\hat{z}+j$ entstandene $i$ nur den ihm unmittelbar roraufgehenden, hinsichtlich der Palatalitätskorrelation neutralen Labial- oder Palatalkonsonanten mouillirit hat, nicht aber den als erstes Glied in der Verbindung stehenden Dental, wodurch dann Wortpaare entstanden wie solggi 'er verschliesst' $\sim$ kol ga $i$ 'es trieft' oder uski 'er führt' $\sim$ muśli i il' wäscht'. In unseren Beispielen bestimmen die (Ippositionen $l-l$ und $s-s$, welchen Stammtypus eine jede Form vertritt. die Palatale jedoch, die in diesem Fall monilliert sind, besitzen auch jetzt kcine distinktive Bedeutung.

3.2.7. Soch einige Worte zu jener bekamnten Erscheimung. 
dass der einem Vordervokal der ersten Silbe und einem nichtmouillierten $s, z$ folgende reduzierte Vokal sowohl im Mokscha als auch im Erza velarer ist als nach den anderen Dentalen. z.B. M ṕiza, Gen. ṕizân, E ṕizę, Gen. ṕizęń '(Vogel)nest' < *pesä; M ṕiksâms, E ṕirsęms, ṕiztsęms 'schlagen, prügeln (M): dreschen (E)' < ${ }^{*}$ peksä-; NB auch M keska, Gen. *keskân' 'die Weichen, die Mitte des Leibes' $<{ }^{*}$ keske, wo sich zwischen $s$ und dem Vokal der zweiten Silbe noch ein Palatal befindet. Die Ursache dieser Erscheinung ist bekannt: die aus vormordw. Zeit stammende phonologische ()pposition $s-s$ ( $>$ zwischen Vokalen $z-z$ ) sollte bestehen bleiben; damit dies gewährleistet war, wurde der nach einem nichtmouillierten Sibilanten stehende vordere Vokal der zweiten Silbe velarisiert, durch welchen Prozess gleichzeitig eine Mouilliermgg des Sibilanten verhindert wurde. Eine ursprïngliche Palatalitït des Vokals der zweiten Silbe wurde bereits angenommen von Palasomen, MSFOu XLI 4, MSFOn XXII S. XIV und Ravila, FUF XX 100, TyK L 385. Ravila nimmt für das Urmordwinische die Entwicklung $*_{\partial}>\dot{\partial}$ $\left(>\mathrm{E}_{\ell}, \mathrm{M} \hat{\partial}\right)$ an. Dem kann man sich vollauf anschliessen. Es muss nur hervorgehoben werden, dass dieser Prozess offensichtlich aus der Zeit stammt, die der sekundären Konsonantenmouillierung im Urmordwinischen voranging, als z.B. di* urmordw. Stämme kul'a-, pora- noch die Gestalt *kula-, *porəhatten. Es liegt kein Grund vor, in der sekundären Mouillierung des anlautenden Konsonanten eine ältere Erscheinung zu sehen als in jener des inlautenden Konsonantismus. Die frühesten rekonstruierbaren mordw. Formen des hier in Frage stehenden Worttypus waren also *piza, *leska usw., aus denen sich, als die späturmordw. Zeit erreicht war, ${ }^{*} \not \dot{p} i z \dot{\partial},{ }^{*} K^{\prime}$ ieskà entwickelt hatten. Im Späturmordw. galb es also droi phonetisch verschiedene reduzierte Vokale: a (Vordervokal), $\vec{a}$ (leicht velarisierter Vordervokal bzw. Mittelvokal) und a (Mittel- oder wahrscheinlicher - Hintervokal, rol. Pilasomen, MSFOu XXII 6; Gramm. mord. jazykov 21).

3.2.8. Die Mouillierung des inlautenden Konsonantismus war also eine Erscheimung, durch die die Vokalharmonie des Urmordwinischen weitgehend gestört wurde. Dies kommt nicht nur in den Auslautrokalen des nichtabgeleiteten Stammes zum 
Ausdruck, sondern ebenso gut in den Vertretern der urmordw. reduzierten Vokale, die in den Flexionsendungen und Ableitunsen enthalten sind. Wemn nämlich des Stamm auf einen mouillierten Konsonanten ausgeht. rertritt der suffixvokal stets ein urmordw. a (a), auch wenn der rorangehende Vokal velar ist. Z.B. E kal-de Abl. $\left(<{ }^{*} k a l-1 / a\right)$, kal-stę $\left(<{ }^{*} k a l-s t a\right)$ Elat. 'Weide', kal-teme Karit. (<*kal-etama) 'ohne Weide; weidenlos', rgl. lial-do Abl. (<*lial-dâ), lial-sto (<*kal-stâ) Elat. 'Fisch', lial-tomo Karit. (<*ial-rtâmâ) 'ohne Fisch; fischlos'.

Der zweite Faktor, durch den die Vokalharmonie wesentlich beeinträchtigt wurde, war das Allgemeinwerden des a der nichtersten Silben, anfangs suffixal, auch in vordervokalischen Stämmen. Näher hierzu s. Ravila, a.a.o. 100-104; Verf., i..i. O. 299 .

3.2.9. In der angeführten Reihe der urmordw. reduzierten Vokale, $\partial, \hat{\partial}, \hat{\partial}$, sind lediglich die horizontalen Artikulationsunterschiede berücksichtigt worden. Es gribt jedoch auch die Iypothese von Nuancenunterschieden in vertikaler Richtung, so dass auch diese Frage zu überprüfen ist. Soweit bekannt sind für das Mokscha solche Differenzen nicht mitgeteilt worden; Paasonen $\%$.B. erwähnt nur a und $\hat{\partial}$, s. a.a.0. 84--86. In den Erza-Dialekten dagegen, die wie das Mokscha reduzierte Vokale kennen, kamn den mordwinischen Forschern zufolge in der velaren Reihe der Wechsel $\hat{a} \sim \hat{?}$ auftreten. Die Vertreter der urmordw. reduzierten Vordervokale werden von ihnen regelmässig als Vollvokale $e$ oder $i$ bezeichnet. Im Dialekt von Naskaftym z.B. begegnet nach Bibin das Vorhältnis kalân 'fischreich' : kämen (kämiñ) 'zehn'. In Paasonens Texten, der seinerzeit dieselbe Mundart untersuchte, finden wir auch einen reduzierten Vordervokil, z.B. kulâs' 'er starb' : kälmaś 'er erfror', s. MSFO LXXXI 523. Es ist schwer zu wissen, ob die unterschiedliche Bezeichnungsart auf eine lautliche Entwicklung im Dialekt zurückgeht oder auf anderen Gründen beruht.

In der letzten Zeit sind mehrere Untersuchungen über die 'rwähnten Erza-Dialekte mit Mokscha-Charakter erschienen. In diesem Zusammenhang kann jedoch nicht näher auf sie eingegangen werden. Wir wollen uns in der Hauptsache darauf

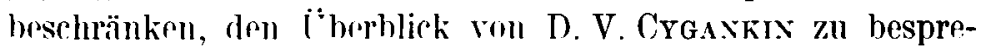


chen, "Opyt klassifikacii erzjanskich govorov mordovskogo Prisurjan, (čerki V 383-394. Wenn wir die zweitrangigen Sonderfällo ïberrehen, teilen sich die Dialokte mit Vokalreduktion in fünf (iruppen: 1. die Gruppe ron sibaljevo, wo nach allen Hintervokilen $(a, u, o)$ der ersten Silbe der reduzierte Vokal ? folgt, z.B. skaḷ̣s' 'Kuh'. kud?̣̂s 'Haus', tšotškị̂s' 'Balken', vgl. auch V. I). OBEukix Očerki III 5; M. S. BIUŠkIN ibid. V 201; 2. die Gruppe von šugurovo, wo nach $a, \ddot{a}, "$ und $i$ ein ? folgt, nach " und $e$ jedoch ein $\hat{a}$, z.B. kandịms 'wegrtragen, führen',

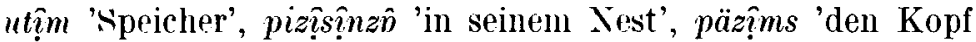
waschen' 〜 śolgâms 'zumachen', lielezânzì 'in seine Zunge'; 3. die (iruppe von Kosogory, wo nach $a$ ein $\hat{?}$ auftritt, nach $o$ aber ein $o$ und nach $u$ ein $u$, z.B. casịla 'entfernt' $\sim$ lovnoma 'das Lesen' $\sim$ udums 'schlafen', rgl. auch Cygankin, Očerki II 438-4+3; 4. die Gruppe ron Parakino, wo nach allen hinteren Vokalen ein ? begegnet oder anstelle dessen der Vollrokal i, z.B. kudissì $\sim$ kudịs $\hat{n}$ 'im Haus'; 5. die Gruppe von Kabajevo, wo $\hat{\partial}$ als velirer reduzierter Vokal steht, z.B. hastâms 'erziehen', uskiâms 'führen', kozâms 'Husten', pizâzânzôn 'in sein Nest'. Da die hier zitierten Beispiele im Original in russischen Buchstaben geschrieben sind, besteht die Möglichkeit, dass trotz der Definitionen der Forscher die reduzierten Vokale in unserer Transliteration nicht immer genau der Aussprache entsprechend bezeichnet sind.

Ergänzend seien noch folgende Angaben hinzugefügt. De! Dialekt ron Naskaftym zeigt densolben Stand wie die Gruppe ron Šugurovo, s. Bibin, Učenyje zapiski 43 S. 56. In der Mundart von Fodorovka ist der reduzierte velare Vokal i.a. durch $\hat{\partial}$ vertreten, nach a jedoch durch $\hat{?}$; nur wenn neben a ein mouillierter Konsonant steht, folgt auch dem a ein $\hat{z}$, z.B. palịms 'brennen (intr.)' 〜 maŕatâms 'hor(hen', tšaŕlâdims 'erkennen', s. Binškin, a.a.O. 221-225. In Dialekt von Kažlỵtha ist nach Palasonen der normale Vertreter des velaren reduzierten Vokals

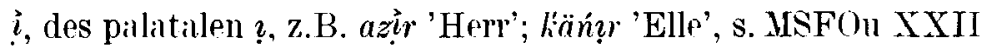
$86-87$.

3.2.10. Die besprochenen Wechsel in den Erza-Dialekten $\hat{\partial} \sim \hat{?}$ und $\partial \sim$ ? können offenbar nicht getrennt werden von den in einigen anderen Erza-Dialekten begegnenden Wechseln 
$o \sim u$ und $e \sim i$, d.h. ${ }^{*} \hat{\jmath}>o,{ }^{*} \hat{?}>u *^{*} \Rightarrow>e,{ }^{*} ?>i$. Mit jenem Wechsel $\hat{a} \sim \hat{?}$, dej im Diallekttypus von s̈ugurovu (vorl. when) rorkommt, ist der Wechsel zu vergleichen. der dem "progressiv-assimilatorischen" (Bubrichs Benemmung) Dialekttyl) eigen ist, wo nach dem $a(a), u$ und $i$ der ersten silbe der nnge Vokal $u$ bzw. $i$ erscheint, nach o und $e$ jedoch " bzw. e. z.B. kasums 'wachsen', putums 'setzen, stollen. legen', simims 'trinken' sornoms 'zittern, bebrul. motems 'gehen', Tedems 'mähen; schiessen, erschiessen'.

3.2.11. Die mordwinischen Forscher sind allgemein der Meinung, die im Erza begrgnenden reduzierten Vokale gingen auf Mokscha-Einfluss zurück, oft zwar auf relativ alten. Auch wenn es sich so verhielte, gehen die Wurzeln des Erza-Wechsols aller Wahrscheinlichkeit nach ins Urmordwinische zurück. Schon Paasonen hielt es für möglich, dass »im gemeinmordvinischen ein derartiger combinatorischer vocalwechsel ausserhalb der ersten silbe bestand, wie jetzt in den meisten ersa-mundarten", s. a.a.O. S. XIII. Dem hat sich Ravila angeschlossen und geäussert, die Wechsel der reduzierten Vokale im Urmordwinischen seien die Voraussetzung gewesen für die Wechselbeziehungen im Erza. "Es ist augenscheinlich, dass die umgebendon Laute auf die unvollkommen artikulierten Vokale in verschiedenen Dialekten leicht auf verschiedene Weise wirken und die so entstandenen Nuancen der reduzierten Vokale sich damn zı deutlich verschiedenen Vokalen "ntwickeln", s. FUF XX 91. Tnterzeichneter nahm seinerseits vor 25 Jahren an, dass im Ermordwinischen nach einem $a, \varepsilon, i$ und $u$ der ersten Silbu dir engeren Varianten der reduzierten Vokale, ${ }^{*}$ ?, ${ }^{*}$ ?, standen und nach einem $o$ und $e$ in der ersten Silbe * $\hat{\partial}, * a$, s. a.a.(). 323-326. Damals war in der Literatur noch nicht rewähnt, dass es auch heute Mundarten rom Šgurovo-Tỵpus gibt, wo eine diesbezügliche Vertretung herrscht (wenigrstens hinsichtlich der velaren reduzierten Vokalr). Nach dor vom Verfasser vorgelegten Erklärung waren die offenen und engen Vokale der ersten Silbe in einer bestimmten Phase des Urmordwinischen gedehnt $\left(^{*} \grave{a}\right.$, $\left.{ }^{*} \dot{\varepsilon},{ }^{*} \grave{u},{ }^{*} i\right)$; als Folge der starken Intensität dieser Vokale kam es dann zu einer Schwächung imı reduzierten Vokal der zweitẹn

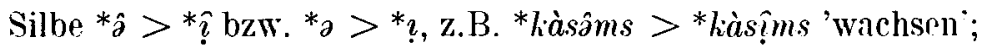


*simams > *simum. 'trinken'. Digegen wilrell o und $e$ in der

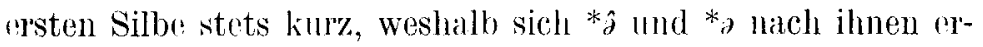
hielten, ohne enger zu werden. Vergloichshalber' wurde damals eine ziemlich ähnliche Erscheinumg aus dem Inarilappischen angeführt. Um irgendeine später progressive Assimilation handelt es sich bei der ${ }^{*} \hat{\jmath}$ - und ${ }^{*}$-Vertretung also nicht.

3.2.12. In einigen Erza-Dialekten, wie in dem des Dorfes ('hlystofki, kam es in der ersten silbe zum Wandel urmordw. ${ }^{*} \varepsilon>e$ (z.B. ked' 'Hand') und andererseits urmordw. $*_{e}>\ddot{a}$, ๘.B. käd' 'Haut'. Die Vokalstruktur der Genitivformen beider' Wörter, keden bzw. läalin, ist in ihrer Gesamtheit sekundär: in der ersten Silbe der ersteren Form haben wir die Entwicklung ${ }^{*} \dot{\varepsilon}>e$ und in der zweiten Silbe $*_{l}>*_{i}>e$; in letzterer wiederum ist das kurze $e$ der ersten Silbe zu ä geworden, in welchem Zusammenhang in der zweiten Silbe die Entwicklung *a $>^{*}$ ? $>i$ eintrat. Im Typus kärl öffnete sich das urmordw. $e$ der ersten Silbe offenbar zu ä, dimit das a anstelle des früheren ein nenes vordervokalisches Patrerhielt. d.h. dep Wandel $e>\ddot{a}$ füllte jene leere stelle, die diss zu e grewordene urmordw. $\varepsilon$ im System hinterlassen hatte. So ist es nur natürlich, dass sich auch beim Typus käarin der Vokil der zweiten silbe zu einem Paar des entsprechenden Vokils vorn Typus kasums entwickelte; die auf alte Voraussetzungon zurückgehende Vokalkombination $a-u(<$ urmordw. $a-?)$ rrhielt somit die spät entstandene Kombination $\ddot{a}-i(<\operatorname{mrmordw}, e-a)$ zur Seite.

In jenem normalen Dialekttyp, auf den die Schriftsprache dos Erza basiert. "rscheint in der zweiten Silbe o nach allen Hintervorkalen und e nach allen Vordervokalen; es handelt sich hier um eine Ausgrleichung, durch die die alten Variantenwechsel verdeckt wurden. Dio Entwicklung dus urmordw. a zu o dürfte durch Vermittlung des libialen Mittelvokals $\dot{o}$ vor sich gegangen sein, den A. A. Šschuarov noch in den Dialekten ron Suchoj Karbulak (z.B. kozoma 'Husten') und Orkino (z.B. olgoza 'sein Stroh') antraf, s. Mordovskij otnografičeskij sbornik 728.

3.2.13. Wir hilben oben den Standpunkt bezogen, dass es in den nichtersten Silben im Urmordwinischen ebenso reduzierte Vokale gab wie hente im Mokscha und in den benachbarten 
Erza-Dialekten. Als erster hatte Šachmatov diesen Gedanken geäussert. Er nahm fül das Urmordwinische zweierlei reduzierte Vokale an: $a$ (bezeichnet durch $\varepsilon$ ) und $\hat{\partial}$ (bezeichnet durch $\alpha$ ), die im Erza zu $e, o, i, u$ usw. geworden sind, s. a.a.0. 761-776. Die eigentlichen Begründungen für diese Theorie brachte Ravila in seiner bemerkenswerten Studie "Über eine doppelte Vertretung des urfinnischwolgaischen $* a$ der nichtersten Silbe im Mordwinischen» (FUF XX). Er wies überzeugend nach, dass der Vertreter des vormordw. ${ }^{*} e$ der nichtersten Silben im Urmordwinischen regelmässig akzentlos und reduziert war. In dieselbe Stellung geriet fast gänzlich das * $\ddot{a}$ und auch ein Teil der *a-Fälle. Reduzierte Vokale im Urmordwinischen hat auch B. A. Serebrennikov angenommen, s. Istoričeskaja morfologija mordovskich jazykov 23. Von den heutigen mordwinischen Forschern vertritt Devajev diese Linie. Zur Unterstützung seiner Auffassung verweist er auf dieselben Umstände wie schon Ravila, nämlich auf den ursprünglicheren Charakter der Mokscha-Akzentuation verglichen mit dem Erza sowie auf den uralten Schwund des Vokals der ersten Silbe, der auch in zahlreichen Erza-Wörtern begegnet, s. Očerki II 272-273.

Die hiermit konkurrierende Erklärung geht aus von den Vollvokalen $i$ und $u$ in den nichtersten Silben des Urmordwinischen, die im Mokscha reduziert worden wären und sich im sich im Erza unterschiedlich entwickelt hätten. Diese Hypothese stammt von Paasonen, s. a.a.9. 96. Ihm hat sich Bubrich in verschiedenen Zusammenhängen angeschlossen (z.B. Ist. gramm. 11), und seinen Spuren folgen fast alle heutigen mordwinischen Forscher. Doch erwïhnt Devajev (a.a.O. 272), auch Bubrich habe in eimem 1947 erschienenen Aufsatz reduzierte Vokale im. Urmordwinischen vorausgesetzt.

3.2.14. Die Frage nach der Chronologie der reduzierten Vokale würde ebenfalls eine eingehende Untersuchung des Vokalismus der mordwinischen Ableitungs- und Flexionssuffixe erfordern. Auch in dieser Hinsicht ist von Ravila wertvolle Vorarbeit geleistet worden, s. i.a.O. 117-120. Er weist auf mehrere Fälle hin, wo das suffixale $a$ der dritten und vierten Silbe mit dem Vertreter des reduzierten Vokals wechselt (z.B. E kulomo, M kulômă 'um zu sterben' $\sim$ E kuloma, M kulâma. 'man muss 
sterben'; E Abl. palomado 'das Brennen' jakamodo 'das Gehen'; ojmamo 'das Ruhen' kuluma 'Tod'; marama, Al)l. maŕamuda 'das Hören' $\sim$ kuluma, Abl. kulumada 'T'od'), und bringt sie mit vollem Grund mit alten Akzentwechseln in Zusammenhang. Bei der Klärung dieser Erscheinungen tritt jener Dialekttyp des Erza in den Mittelpunkt der Aufmerksamkeit. den Bubrich - vom Standpunkt der historischen Entwicklung zweifellos wieder irreführend - "regressiv-assimilatorisch" nennt. Charakteristisch für diesen Inialekttypus ist, dass in der zweiten silbe $u, i(i)$ erscheint, wenn in der dritten Silbe $a, u$. $i$ steht, jedoch $o$, $e$, wenn auch in der dritten Silbe dieselben halbengen Vokale auftreten, z.B. kunsulan 'ich höre zu', liunsuli 'er hört zu', kunsulutano 'wir hören zu' kunsoloms 'zuhörrn'. kunsolok 'höre zu!', kunsolodo 'hört zu!', s. Bubrich, a.i.(). 9. Eine solche Wechselerscheinung hätte ummöglich in riner sprache wie dem Erzamordwinischen entstehen können, wo die Akzentunterschiede zwischen den Silben ausserordentlich unbestimmt sind. Hier wird deutlich in eine Zeit verwinsen, da die Vollvokale $a$ und $i(i)$ der dritten und vierten Silbe cinen so starken Akzent trugen, dass die ihnen vorangehenden reduzierten Vokale zu ${ }^{*} ?$ und ${ }^{*} ?(>\mathrm{E} u, i)$ abgeschwächt wurlen, während $*_{\partial}$ und $*_{\partial}(>\mathrm{E} o, e)$ unverändert erhalten blirben. wenn in den nichtersten silben nur reduzierte Vokile standen.

3.2.15. Ausser den zugunsten der Theorie, die urmordw. reduzierte Vokale voraussetzt, bereits angeführten Gesichtspunkten kamn wenigstens noch auf folgendes aufmerksam gamacht werden. Würde die Auffassung von Paasonen und Bubrich zutreffen. hätte sich vormordw. *hulke- im Crmordw. entwickelt zu *kolgi-, rormordw. *sulle- aber zu *solgu-. Der' Wandel $*_{e}>i$ ist zwar natürlich, doch kann dasselbe kaum von der Entwicklung ${ }^{*} e>u$ gresigt werden. Phonetisch nicht ohne weiteres nachvollziehbar erscheint ferner der Wandel ${ }^{*} u j>i$, der im Typus solgi 'er schliesst' cingetreten wäre, falls dieser tatsächlich auf die Form * solgu-j zurückginge. Wenn dagegen angenommen wird, dass sich das *e der zweiten Silbe sowohl im Fall *liullie- als anch * sulle- erst zu dem reduzierten Vordervokial a rntwickelte, der im letzteren Wort zu a wurde, 
weist der Verlauf der Entwicklung nichts phonetisch Befremdendes auf: die Distanz zwischen $\partial$ und $\hat{\partial}$ ist $u m$ ein Beträchtliches kleiner als die phonetische Differenz zwischen $i$ und $u$; ausserdem ist der Wandel ${ }^{*} \hat{\partial} j>i\left({ }^{*}\right.$ śolg $\left.\hat{\partial}-j>s^{\prime} \circ l g i\right)$ wegen der Illabialität und der unvollkommenen Artikulation des dem $j$ vorangehenden Lautes viel wahrscheinlicher als ein Wandel $* u j>i$.

3.2.16. Noch cinige Worte zu den allgemeinen Voraussetzungen für ein Erscheinen von reduzierten Vokalen in nichtersten Silben. Im Mokscha begegnet $\partial$ und $\hat{\partial}$ ausser im Inlaut auch im Auslaut des Wortes, falls nicht unmittelbar eine Pause folgt. Vor der Pause wird statt a ein $\varepsilon$ (> dial. $e$ ) gesprochen und statt $\hat{\partial}$ ein $a$; diese Vollvokale sind stets akzentlos. Im Dialekt von Gorodišče stehen die der diesbezüglichen Pause vorangehenden Vokale nach Lomakina vqualitativ dem (a) oder (e) nahe" (Očerki IV 303) und unterscheiden sich also irgendwie von den auf urmordw. Vollvokale zurückgehenden a und $\varepsilon$. Auch Tultajev sagt, der reduzierte Vokal stehe im absoluten Auslaut nach wharten" Konsonanten »dem a nahe». Er fügt hinzı: "В конце слова редуцированный в чистом виде произносится в Темниковском районе, частично в Атюрьевском и ІІудоманском районах", s. a.a.0. 70. Einige Beispiele: Sredne-Vad ludne 'meine Häuser' kudñ palst 'meine Häuser verbrannten', lama 'viel' 〜 lamo lomaí 'viele Leute' (der reduzierte Vokal erscheint in kontinuierlicher Rede vor' einem Wort mit konsonantischem Anlaut, s. Devajev, a.a.(). 280-281); Temjaševo $p i \cdot \gamma_{g} \varepsilon$ 'Fuss' $\sim p i \cdot l g \hat{z}$ ki 'Fussspur',

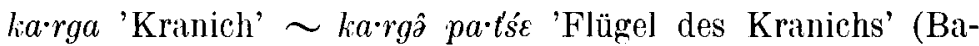
buškina, Očerki IV 48); Gorodišče nile 'vier' nilə koft 'vier Monate', tusta 'dicht' tustâ viŕ 'ein dichter Wald' (Lomakina, a.a.0. 303-304); Palajevka $t u \cdot m a ̈$ 'wir kamen' $\sim t u \cdot m o k u \cdot d u$ 'wir kamen nach Hause', $t u \cdot m a$ 'Eiche' $\sim t u \cdot m \tilde{o}$ sud 'Eichenrinde' (V. HallaP CSIFU I 163); Rybkino-Mamolajevo pilä 'Ohr' 〜 pil’ potänää 'Ohrläppchen', koza 'wohin' kozâ mol'at 'wohin gehst du ?' (s. Lipatov, SFU V 180, wo sich auch Hinweise auf die entsprechende Erscheinung in einigen anderen Mundarten finden).

Im Mokscha haben sich die akzentlosen Vollvokale im Wort- 
auslaut offenbar folgendermassen entwickelt: $*_{\partial}>*_{3}>\varepsilon$; ${ }^{*} \hat{\partial}>{ }^{*} \hat{n}\left(\right.$ bzw. $\left.{ }^{*} p\right)>a$, d.h. reduzierte, aber ntwas offenere Vokale als es die ursprïnglichen sind, bildeten auf dem Woge zur heutigen Vertretung eine Zwischenphase. Dieser Prozess wird deutlich sichtbar in zahlreichen Erza-Mundarten mit Mokscha-Färbung, wo noch heute gerade ein solcher offener reduzierter Vokal im Wortauslaut begegnet. Reduziert haben die Forscher zwar generell nur den Vertreter des urmordw. velaren a bezeichnet. Nach der Definition von Cygankin handelt es sich um einen reduzierten halboffenen (Očerki I 316) oder offenen (ibid. V 394) Mittelvokal, am nächsten wohl $\hat{p}$. Beim Transliterieren von Belegen aus einigen anderen Quellen dürfte, das Zeichen $v$ angebrachter sein. Einige Beispiele: SabajevoKačelaj štapp 'nackt', pondp 'Pud' (Obedkin, Očerki III 5); Davydovo sardn 'Stachel', tšarn 'leer' (Obedkin, ibid. II 41); Šlugurovo latkp 'Schlucht', tšotšln 'Balken' (Cygankin, ibid. I 316-317); Kosogory pulv 'Schwanz', vadrastôn Adv. 'gut' (Cygankin, ibid. II 442); Parakino kunsị̂l l̂dô 'hört zu!' (Cygankin, ibid. V 390); Kabajevo kunsâlâd̂̂ id. (Cygankin, a.a.O. 391); Naskaftym kardp 'Stall', mäzzdo Abl. 'was' (Bibin, Učenyje zapiski 43 S. 53). Im Dialekt des in der Baschkirischen R.publik gelegenen Dorfes Fedorovka steht nach Biuškin im Wortauslaut statt $\hat{\partial}$ ein Vokal, der in seiner Klangfarbe dem $e$ ähnelt, jedoch ein offenerer, gemischter Vokal ist, also wohl $\hat{3}$, z. B. parḡ̄ 'Korb', tust̂ิ 'dick', s. Očerki V 207.

Ausserdem gibt es im Erza Dialekte, wo â im Wortauslaut vor einer Pause zum Vollvokal a geworden ist wie im Mokscha, z.B. Kaljajevo, Kažlytka karda 'Stall' (Paasonen, a.a.O. 86 -87), Suchoj Karbulak, Orkino huda 'Haus' (Šachmatov, a.a. (). 722), Drakino kuda id. (A. V. JAkUškix Očerki I 205). Dê wortauslautende Vertreter des urmordw. *a ist auch in fast all den Dialekten als Vollvokal bezeichnet, die als Vertreter von urmordw. *â einen reduzierten Vokal kennen, z.B.SabajevoKačelaj elde 'Stute', Darydovo rete 'fünf', Š́ugurovo päkä 'Bauch', Kosogory sịrä 'alt', pitši 'Kiefer', Naskaftym kiärilä 'Filz'. Im Dialekt von Fedorovka ist auch das *a im Wortaus-

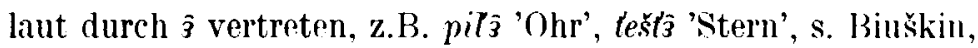
a.a.O. 208. 
4.1. Wie sind die reduzierten Vokale im Mordwinischen phonologisch einzuschätzen? Die meisten mordwinischen Forscher haben in ihren Dialektuntersuchungen die Vokale a und a im Mokscha und in einigen Erzi-Dialekten als Allophone ein und desselben, meist als Mittelvokal charakterisierten und z.B. mit dem Buchstaben $z$ bezeichneten reduzierten Phonems brtrachtet; die Voraussetzungen für deren Auftreten sind jedoch nicht näher analysiert worden. Auch Hallap sieht die Vokalreduktion als distinktive Erscheinung, meint jedoch, a und $\hat{a}$ (= Hallap $\tilde{o}$ ) seien aus praktischen Gründen als verschiedene Phoneme zu deuten. Wenn man von einem einzigen Phonem a ausgeht, wird seines Erachtens die Beschreibung von dessen phonetischen Eigenschaften und von der Distribution der einzelnen Allophone recht kompliziert, s. a.a.O. 163.

Anderer Meinung ist KáRoly RÉdeI, der in NyK LXX 383 -386 ausführt, die reduzierten Vokale des Mokscha seien lediglich in unbetonter Stellung auftretende Allophone der Vollvokale $e, i, o, u$. Dieselbe Behauptung wiederholt er in ALH XX 457. Beidemale polemisiert Rédei gegen andere Forscher (Devajer, W. Veenker), die die reduzierten Vokale als von den Vollvokalen getrennte Phoneme angesehen haben. Bei der Behandlung des Erza-Dialekts von Drakino, wo in den nichtersten silben reduzierte Vokale begexnen, vertritt auch Jakuškin dif Ansicht, der reduzierte Vokil $\mathbf{z}(=\hat{\jmath}$; z.B. kulâs' 'er starb') sei kein selbständiges Phonem, sondern nur eine Variante des Phonems o, s. Očerki I 201, ibid. III 53.

Bei der Untersuchung dieser Fragen müssen wir uns priktisch auf das Mokscha beschränken, denn soviel neues und interessantes Material auch in den Abhandlungen über die ErzaDialekte mit Mokscha-Färbung enthalten ist, hat man wohl in keiner einzigen davon das Thema vom phonologischen Standpunkt aus erschöpfend behandelt, so dass diese Materialien nicht zu sicheren Schlussfolgerungen berechtigen.

4.2. In der ersten $S$ ilbe ist das halbenge $e$ und 0 stets akzentuiert. Es ist deshalb unmöglich, von Allophonen des $e$ und $o$ zu sprechen, die in akzentloser Stellung auftreten. (Das oben Gesagte gilt nicht für russische Wörter, die im Schrift-Mokscha und vielleicht anch in den Mundarten begegr- 
nen, sich dem Lautsystem der Sprache nicht angeglichen haben und in deren erster Silbe auch akzentloses $e$ und $o-$ wenigstens in der Schrift - in Frage kommen, z.B. neчá.lb 'Sorge', nopóz 'Schwelle', s. Potapkin-Imjarekov, Mokšansko-russkij slovar' 206,215 .) So gilt z.B. in Beziehungen wie maźa ra 'wieviel' $\sim$ me.źk 'was' (val. oben Punkt 2.1.2.) und kâda. 'wie; wenn; als' $\sim k \omega \cdot d a$ id. (vgl. Punkt 2.1.3.) nicht der Wechsel von Allophonen, wie Rédei grlaubt, sondern ron Phonemen. Das a und $\hat{a}$ dieser Beispiele sind ebensolche Phoneme wie die bald unten zur Sprache kommenden reduzierten Vokale.

Komplizierter ist die phonologische Analyse solcher Wechsel, all denen eimmal a und $a$ beteiligrt sind, zum andern die engen Vollvokale $i$ und $u$. Hinsichtlich der Akzentverhältnisse sind alle diese Vokale insofern miteinander verorleichbar, als sie vor einem urmordw. a der zweiten Silbe akzentlos sind. Die Vokalreduktion ist in akzentloser Stellung eingetreten, doch ist, wio Cudajeva sagt, "der reduzierte Laut in der späteren Entwicklung infolge spezieller phonetischer Assoziationen auch in akzentuierte Stellung eingedrungen, im allgemeinen jedoch nur in der ersten Silbe des Wortes", s. Zapiski 18 S. 226. Vgl. auch oben Punkt 2.2.5. Solche Fälle sind etwa: kôेtmôldâms 'sich schlängelı: wimmeln'; kầrliśams frequl. 'schaben' ( $\sim$ E kurkśems, hirk'sems) ex analợia kârga·ms 'schaben' ( E korgams, kirgams); pầl'ńams tr. frequ. 'brennen' ( $\sim$ E pultnems) ex analogia pâl'ta.ms 'brennen' ( $\sim$ E pultams). Allgemein haben die mordwinischen Forscher demu auch aus diesem Grund den Standpunkt bezogen, dass der reduzierte Vokal ein selbständiges Phonem ist und keine Variante von $i$ oder $u, s$. z.B. framm. mord. jazykov 30; Cudajeva, a.a.O.

Wer das Mokscha nicht selbst gehört hat, kann den wirklichen Charakter der in den verschiedenen Quellen genannten Wechsel $a \sim i$; $\hat{a} \sim u$ u.dgl. nicht so leicht beurteilen, allein schon ans dem Grund, weil es in der Intensität der Vokalreduktion nach Paasonen Nuancenunterschiede gibt (vgl. oben Punkt 2.2.5.), und zweitens deshalb, weil die Vertretung der einzelnen Dialekte nicht einheitlich ist. Zumindest in Dialekten wie Sredne-Vad und Gorodišče, aus denen u.a. das Wortpaar purda.ms 'drehen, winden' $\sim$ pârda'ms 'verstecken, verbergen' 
(s. Devajev, Očerki II 264; Lomakina, ibid. IV 291) angeführt wird, kann $\hat{a}$ nicht das Allophon von $u$ sein. In der gleichen Weise sind $\hat{\partial}$ und $a$ ron $u$ und $i$ getrennte Phoneme in allen anderen Dialekten, wo die Reduzierung des akzentlosen engen Vollvokals nur sporadisch reschehen ist (dazu s. oben 2.2.5. und 2.3.3.). Falls es Mundarten gäbe, wo in der akzentuierten Silbe stets $i, u$ steht, in der akzentlosen immer $\partial, \partial$ (z.B. ki rodzms 'halten' $\sim$ kard $a \cdot n$ 'ich halte'; :ku·lâms 'sterben' $\sim$ kâla.n 'ich sterbe'), dann wären dort die engen Vollvokale und die reduzierten Vokale im Vokalsystem der ersten Silbe tatsächlich Allophone voneinander. In jedem Fall sind jedoch auch ill solchen Mundarten die reduzierten Vokale der nichtersten Silben ron den diesbezüglichen Vollvokalen getrennte Phoneme (s. unten), welcher Umstand bei der phonologischen Gesamtdarstellung des Vokalismus der betreffenden Dialekte zu berücksichtigen ist.

4.3. In den n i c h t e r st e n s i l b e n des Mokschit-Mordwinischen begegnen $e$ und $o$, die auch in dieser Silbenstellungr akzentuiert sind, lediglich in jungen Lehnwörtern sowie in Komposita, wie von zahlreichen Forschern festgestellt wird, s. Devajev, a.a.O. 278; Babuškina, a.a.O. 44; Azrapkin, Očerki IV 255; Lomakina, a.a.O. 301-302; Lipatov, a.a.O. 176. (Hiel' sind nicht solche Dialekte gemeint wie z.B. der von SredneVild. wo das anderwärts auftretende $\varepsilon$ zu $e$ geworden ist, z.B. ale Mann' < alc s, Devajev, a.a.O. 269.) Rédei sieht die Dingro folgendermassen: "Das reduzierte a hat nach einem normalen Konsonanten den Lautwert eines $o$, nach mouillierten Konsonanten sowie nach $\check{s}, \not{z}$ und $\check{c}$ den eines $e »$,' s. NyK LXX 384. Ebenso wenig wie in der ersten Silbe können die akzentlosen $\partial$ und $\hat{\partial}$ in den nichtersten Silben Allophone der akzentuierten $e$, $o$ sein, die in dieser Stellung in echten (nichtzusammengesetzten) mokschanischen Wörtern nie vorgekommen sind. Im SchriftMokscha wird zwar z.B. cúмомс 'trinken', ша́чемc 'geboren werden' geschrieben, doch sind $o$ und $e$ nur Grapheme der reduzierten Vokale (vgl. z.B. Tultajev, a.a.O. 74-76; Gramm. mord.

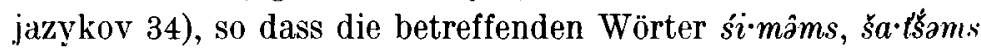
ausgesprochen werden sollen.

Auch sind a und $\hat{a}$ nicht die Allophone von $i$ und $u$. Devajer 
erläutert diesen Umstand u.a. an den folgenden Wortpaaren: $l \varepsilon \cdot d \partial t^{\prime}$ 'du mähtest' $\sim l \varepsilon \cdot d i t$ 'des Mähers'; $p u \cdot l a \hat{s}$ 'der Schwanz' $\sim p u \cdot l u s$ 'der mit einem Schwanz Ver'sehene', s. a.d.(). 264.

4.4. Die inlautenden $\partial$, $\hat{\partial}$ könnon i m A us la ut mit anderen Latuten wechseln, die i.a. als Vollvokile $\varepsilon$, a charakterisiert sind, z.B. iele Nom. ielań Gen. 'Dorf'; ula Nom. ulân Gen. 'Kinn', vgl. oben Punkt 3.2.16. Insofern diese auslautenden Vokale qualitativ identisch sind mit dem auf einen urmordw. Vollvokal zurückgehenden $\varepsilon$ und a (z.B. al $\varepsilon$ 'Mann', t'sora 'Bursche'), haben sie als dieselben Phoneme wie diese zu gelten. Dabei macht sich in einigen Fällen dif distinktive Funktion des Akzents im Mokscha geltend, d.h. sein Charakter eines suprasegmentalen Phonems: $t u \cdot m a$ 'sich begeben' (<tu'mâ) $\sim$ tuma. 'man muss sich begeben', vgl. Hallap, a.a.(). 166. Zı stark in den Fesseln lauthistorischer Betrachtungsweise befangen, haben einige Forscher die mit a und $\hat{a}$ wechselnden auslautendon $\varepsilon$ und $a$ als Varianten oder Realisationen eines reduzierten Phonems gedentet, auf welchen Irrtum I. P. GRUzov in SFU V 156 aufmerksam gemacht hat. Wenn sich wiederum die in Frage stehenden auslautenden Vokale irgendwie von den Vertretern des urmordw. Vollvokials, $\varepsilon$ und $a$, unterscheiden (was in einigen Dialekten der Fall sein dürfte, rg̨l. oben Punkt 3.2.16.), und in keiner anderen Stellung erscheinen, dann sind sie tatsächlich nur Allophone eines reduzierten Phonems. Die in einigen Erza-Dialekten mit inlautendem $\hat{z}$ wechselnden auslautenden $n, \hat{n}, \hat{3}$ (vgl. Punkt 3.2.16.) sind niltürlich als Allophone von $\hat{\partial}$ zu betrachten.

4.5. Zusammenfassend kann gesagt werden, dass die reduzierten Vokale im Mokscha nicht als Allophone von $e$ und o erklärt werden können. Das gilt allgemein genommen auch für die Beziehung zwischen reduzierten Vokalen und $i$ und $u$. Falls man sich bei der Untersuchung nur auf den Vokalismus der ersten Silbe beschränkt, existieren vielleicht auch solche Dialekte, wo die reduzierten Vokale als akzentlose Allophone von akzentuiertem $i$ und $u$ ausgelegt werden könnten.

Das dem Verfasser zur Verfügung stehende Material reicht nicht aus zur Erläuterung der Frage, ob die Vokale a und $\hat{\partial}$ im Mokscha allgemein Allophone desselben Phonems oder aber 
gotrennte Phoneme sind, so dass wir hier nicht dirauf ringehen.

5.1. Hinsichtlich des für das Urmordwinische anzunchmenden Wortakzentes war man in der letzten Zeit weitgehend einer Meinung. Die Auffassung von Sachmatov, wonach der Akzent stets auf der crsten Silbe lag (a.i.(). 761), ist ihrer offensichtlichen Einseitigkeit wegen abgelehnt worden, s. Paasonen, FUF XIII, Anz. 23; Ravila, ibid. XX 90-91. Herrschend wurde die Meinung von Paasonen (MSFOu XXI1 119), Ravila (a.a.O. 90-91, 105, 109-110) und Bubrich (u.a. Ist. gramm. 36-38), dass die Akzentuation im Urmordwinischen wechselnd war. ${ }^{1}$ Paasonen und Bubrich vertraten die Ansicht, der Akzent im Mokscha (vgl. oben Punkt 2.1.2.) habe direkt die urmordw. Vertretung erhalten; auch die heutigen mordwinischen Forseher haben sich dieser Auffassung allgemein angeschlossen. Die Akzentuation im Späturmordwinischen hat tatsächlich der des Mokscha entsprechen kömnen, wenn jedoch die Beziehungen zwischen Frühurmordwinisch und Mokscha vorolichen werden, darf man mit Ravila "nur sagen. dass sich das Prinzip selbst fortgeerbt hat», s. a.a.(). 109. In Frühurmordwinischen gringr der Hauptakzent vom Vertreter des rormordw. kurzen engen Vokals dor ersten Silbo äber auf das a der zweiten Silbe, wio Ravila nachwies, eventuell in der ersten Phase der Entwicklung auch auf * $\ddot{a}$, vgl. oben 2.3.1. Dies betrifft die zweisilbigen Wortstämme; bei mehrsibigen Ableitumgen wird die Fragr etwas komplizierter und hart noch weiterer Untersuchungen.

5.2. Eine Akzentuation vom Mokscha-Typ hat für das Urmordwinische früher auch V. I. LצTKIx angenommen, der sich noch in einer 1966 veröfentlichten Arbeit vorbehaltlos der Auffassung von Bubrich anschliesst und hinzufügt: "davon zeugen zahlreiche Fälle von Vokalausfall in der ersten silbe jener Wörter, denen in Mokšanischen oder in einzelnen Dialekten des Erźanischen Wörter mit $u, i$, a in der ersten silbe und (gewöhnlich) mit dem Vokal $a$ in der zweiten oder den folgen-

1 Paasonen wollte die Frage der urmordwinischen Akzentuation in den später geschriebenen Zusätzen zu seiner I)issertation (a.a.O. S. IX, XIV) offenlassen, kehrte aber in FCF XIII, Anz. 23 zu seinem ursprünglichen Standpunkt zurück. 
den silben entsprechen, z.B. erź. šlams 'schütteln', mokš. suliams; erź. p'a 'Kopf', folkl. pir̆a usw.», s. UAJb 37 S. 60. Der 1970 erschienene Aufsatz »Problema leksičeskogo udarenija $\checkmark$ finno-ngorskich jazykach" (ALH XX 245-263) zeigt jedoch, dass Lytkin neue Bahnen eingreschlagen hat. Er nimmt an, in der finnisch-lugrischen Ursprache hätte ein völlig freier Akzent treherrscht, der sich gerade u.a. im Elza erhalten habe, s. a.a.o. 260. Der Gedanke an einen freien Akzent in der fiu. Ursprache oder cinen stattdessen eventuell vorhandenen syntagmatischen Akzent taucht bei Lytkin zwar schon in der Arbeit "Istoričeskij. vokalizm permskich jazykov» S. 233-235 (1964) auf. Vielleicht wurde er damals beeinflusst von Palasonens ähnlichen Ideen (MSFO XXIIS. IX), die dieser selbst zunindest für das Mordwinische später verwarf. Völlig unmotiviert ist Paasonens Auffassung, die Beziehungen im Tscheremissischen würden die Hypothese vom freien Akzent stützen. Äusserst fragwürdig ist lerner die Bewciskraft der von ihm genannten Akzentverhältnisse im Südostjakischen, zumal in der Lautstruktur der ostjakischen Wortstämme nunmehr Züge nachgewiesen wurden, die offensichtlich auch den urostjakischen Akzent erhellen und rrleichzeitig in erstaunlichem Masse an die Beziehungen im Frülurmordwinischen erimnerı: fiu. $a$. $\ddot{a}$ ist im Ost.jakischen oft im Inlant erhalten und in allen zwcisilbigen $a^{-}, \ddot{a}$-stämmigen Verben (ihre Anzahl beträgt ca. 50) ist der Vokal der ersten silbe reduziert, s. GenT SAUER (sIFU I $459-461$. I Iytkins Folgerung über einen völlig freien Akzent, der permischerseits gehrrescht und sich teils in den komi-syrjänischen Dialehten bewahrt hätte (ALH XX 256, 260), steht in Widerspruch zu dem von ihm angeführten Material und zu seinen früheren vorzüglichen Akzentuntersuchungen. In der Arbeit "Ob udarenii צ komi-permjatskom jazyke» (Trudy instituta jazykoznanija I 107-119) hat Lytkin detailliert die Entwicklung der permjakischen Akzentverhältnisse dargelegt und festgestellt, dass den ältesten Stand jene komi-jaźvinschen und ońkovskischen Dialokte vertreten, deren Wortakzent (in nichtzusammengesetzten Wörtern) genau nach der Vokalstruktur der Wörter bestimmt wird, s. a.a.0. 117. Auch hinsichtlich des Mordwinischen ist Lytkin nun ein offensichtlicher Lapsus unterlaufen; er vergass, jene 
Zahlreichen Einzelheiten der Lautstruktur im Erza- und Mokscha-Mordwinischen zu berücksichtigen, die die Annahme eines klaren Wortakzentes im Urmordwinischen verlangen (vgl. oben $3.2 .13,3.2 .14$. und 3.2.15.) und unter denen die reduzierten Vokale wertvollste Beweisstücke bilden.

5.3. T. A. Isajeva hat in der Arbeit $" O b$ udarenii $v$ erzjamordovskom jazỵke" (Mord. naučno-issl. inst.: Trudy XXIX 264-274) die Frage behandelt, wodurch die dynamische Akzentuation des Urmordwinischen zu einer völlig unbestimmten im Erza hat werden können. Sie zitiert u.a. eine nur im Manuskript vorhandene Untersuchung von J. D. Polivaxov, wonach der diesbezügliche Prozess ein Ergebnis des Aufeinandertreffens von Erza und Russisch wäre. Mit vollem Grund kann man jedoch wie Isajeva fragen, weshalb der Kontakt mit dem Russischen gerade den Akzent im Erza gestört hätte, da doch für das Russische kein solcher Einfluss auf irgendeine ander Nachbarsprache nachgewiesen ist. Interessant ist in diesem $\mathrm{Zu}$ sammenhang auch die Beobachtung von Paasonen: "Es mag hier erwähnt werden, dass schriftkundige ersaner im dorfe Maresevo russische texte, die ich ihnen vorlegte, ohne schwierigkeit richtig und übereinstimmend accentuirten, dagegen wenn ich sie mordvinische texte mit accentzeichen versehen liess, beständig dabei auseinander gingen", s. a.a.0. 8, Anm.

()b hinter der Entwicklung der Akzentverhältnisse im Erza nicht letzten Endes die auf eigenem Boden entstandene Tendenz steht, eine möglichst grosse Harmonie zu erzielen zwischen dem Vokalismus der ersten Silbe und der nichtersten Silben? In jenen Erza-Mundarten, die reduzierte Vokale kennen, erinnert auch die Akzentuation an die im Mokscha. In einem Dialekttypus z.B., wo die reduzierten Vokale zu Vollvokalen geworden sind und auch in nichtersten Silben die Vokale $a, e$ $(e), i(i), o, u$ begegnen, ist im Vokalismus - unabhängig von der Silbenstellung - eine vollständige qualitative Übereinstimmung erzielt. Dieselbe Tendenz hat sich z.B. auch in Finnischen und Ungarischen realisiert, obwohl sich ebenfalls in diesen Sprachen der Vokalbestand der ersten und nichtersten Silben ursprünglich stark voneinander unterschied. Nur in einer Hinsicht nimmt im Finnischen und Ungarischen die erste Silbe 
hinsichtlich der anderen Silben eine Sonderstellung ein: sie hat ihren ursprünglichen Hauptakzent behalten. Das Streben nach Gleichgewicht ist im Erza noch einen schritt weiter gogangen: die nichtersten Silben sind anch für die Akzentuation gleichwertig geworden mit der ersten Silbe. Diese Ausgleichung hat besonders dadurch gefördert werden können, dass einer der Vokale der nichtersten Silben, nämlich $a$, schon von jeher den Hauptakzent tragen konnte und in den Flexionssuffixen sein ursprüngliches Auftreten durch Analogic beträchtlich erweitert hatte.

ERKKI ITKONEN 